DESY 16-134

July 2016

\title{
Rare B-Meson Decays at the Crossroads ${ }^{*}$
}

\author{
Ahmed Ali* \\ Deutsches Elektronen-Synchritron DESY \\ D-22607 Hamburg, Germany \\ *E-mail: ahmed.ali@desy.de
}

\begin{abstract}
Experimental era of rare $B$-decays started with the measurement of $B \rightarrow K^{*} \gamma$ by CLEO in 1993, followed two years later by the measurement of the inclusive decay $B \rightarrow X_{s} \gamma$, which serves as the standard candle in this field. The frontier has moved in the meanwhile to the experiments at the LHC, in particular, $\mathrm{LHCb}$, with the decay $B^{0} \rightarrow \mu^{+} \mu^{-}$at about 1 part in $10^{10}$ being the smallest branching fraction measured so far. Experimental precision achieved in this area has put the standard model to unprecedented stringent tests and more are in the offing in the near future. I review some key measurements in radiative, semileptonic and leptonic rare $B$-decays, contrast them with their estimates in the SM, and focus on several mismatches reported recently. They are too numerous to be ignored, yet, standing alone, none of them is significant enough to warrant the breakdown of the SM. Rare $B$-decays find themselves at the crossroads, possibly pointing to new horizons, but quite likely requiring an improved theoretical description in the context of the SM. An independent precision experiment such as Belle II may help greatly in clearing some of the current experimental issues.
\end{abstract}

Keywords: Standard Model, Flavour Physics, Rare B Decays, LHC, Anomalies.

\section{Introduction}

The interest in studying rare $B$ decays is immense. This is due to the circumstance that these decays, such as $b \rightarrow(s, d) \gamma, b \rightarrow(s, d) \ell^{+} \ell^{-}$, are flavourchanging-neutral-current (FCNC) processes, involving the quantum number transitions $|\Delta B|=1,|\Delta Q|=0$. In the $\mathrm{SM}^{1}$, they are not allowed at the tree level, but are induced by loops and are governed by the GIM (Glashow-Iliopoulos-Maiani) mechanism ${ }^{2}$, which imparts them sensitivity to higher masses, $\left(m_{t}, m_{W}\right)$. As a consequence, they determine the $\mathrm{CKM}^{3}$ (Cabibbo-Kobayashi-Maskawa) matrix elements. Of these, the elements in the third row, $V_{t d}, V_{t s}$ and $V_{t b}$ are of particular interest. While $\left|V_{t b}\right|$ has been measured in the production and decays of the top quarks in hadronic collisions ${ }^{4}$, the first two are currently not yet directly accessible. In the SM, these CKM matrix elements have been indirectly determined from the $B^{0}-\bar{B}^{0}$ and $B_{s}^{0}-\bar{B}_{s}^{0}$ mixings. Rare $B$-decays provide independent measurements of the same quantities.

In theories involving physics beyond the SM (BSM), such as the 2-Higgs doublet models or supersymmetry, transitions involving the FCNC processes are sensitive to

* To be published in the Proceedings of the Conference on New Physics at the Large Hadron Collider, Nanyang Technological University, Singapore. 29 February - 4 March, 2016. 
the masses and couplings of the new particles. Precise experiments and theory are needed to establish or definitively rule out the BSM effects. Powerful calculation techniques, such as the heavy quark effective theory (HQET) 5 and the soft collinear effective theory (SCET) $)^{6 / 8}$ have been developed to incorporate power $1 / m_{c}$ and $1 / m_{b}$ corrections to the perturbative QCD estimates. More importantly, they enable a better theoretical description by separating the various scales involved in $B$ decays and in establishing factorisation of the decay matrix elements. In exclusive decays, one also needs the decay form factors and a lot of theoretical progress has been made using the lattice $\mathrm{QCD}^{9}$ and $\mathrm{QCD}$ sum rule techniques ${ }^{10}$, often complementing each other, as they work best in the opposite $q^{2}$-ranges. It is this continued progress in QCD calculational framework, which has taken us to the level of sophistication required to match the experimental advances.

In this paper, I review what, in my view, are some of the key measurements in the radiative, semileptonic and leptonic rare $B$-decays and confront them with the SM-based calculations, carried out with the theoretical tools just mentioned. However, this is not a comprehensive review of this subject, but the hope is that the choice of topics reflects both the goals achieved in explaining some landmark measurements and focus on open issues. In section 2 , I review the inclusive and some exclusive radiate rare $B$-decays. There are no burning issues in this area - at least not yet. In section 3 , the corresponding inclusive and exclusive semileptonic decays are taken up. Again, there are no open issues in the inclusive semileptonic decays, but experimental precision is limited currently, which is bound to improve significantly at Belle II. There are, however, a lot of open issues in the exclusive semileptonic decays, in particular in $R_{K}$, the ratio of the decay widths for $B \rightarrow K \mu^{+} \mu^{-}$and $B \rightarrow K e^{+} e^{-}$, hinting at the possible breakdown of lepton universality, the linchpin of the SM, reviving the interest in low-mass leptoquarks. One should also mention here similar issues in tree-level semileptonic decays, such as $R_{D}^{\tau / \ell}$ and $R_{D^{*}}^{\tau / \ell}$, the ratios involving the decays $B \rightarrow D^{(*)} \tau \nu_{\tau}$ and $B \rightarrow D^{(*)} \ell \nu_{\ell}(\ell=e, \mu)$. There are also other dissenting areas, which go by the name of $P_{5}^{\prime}$-anomaly, which is a certain coefficient in the angular description of the decay $B \rightarrow K^{*} \mu^{+} \mu^{-}$, which presumably need a better theoretical (read QCD) description than is available at present. They are discussed in section 3. In section 4 , we discuss the CKM-suppressed $b \rightarrow d \ell^{+} \ell^{-}$ decays, which is a new experimental frontier initiated by the LHCb measurements of the branching fraction and the dimuon invariant mass distribution in the decay $B^{ \pm} \rightarrow \pi^{ \pm} \mu^{+} \mu^{-}$. Finally, the rarest $B$ - and $B_{s^{-}}$decays measured so far, $B \rightarrow \mu^{+} \mu^{-}$ and $B_{s} \rightarrow \mu^{+} \mu^{-}$, are taken up in section 5. Current measurements also show some (mild) deviations in their branching ratios versus the SM. A representative global fit of the data on the semileptonic and leptonic rare $B$-decays in terms of the Wilson coefficients from possible new physics is shown in section 6 . Some concluding remarks are made in section 7 . 


\section{Rare Radiative $B$-decays in the SM and Experiments}

In 1993, the CLEO collaboration at the Cornell $e^{+} e^{-}$collider measured the decay $B \rightarrow K^{*} \sqrt{14}$, initiating the field of rare $B$-decays, followed two years later by the measurement of the inclusive decay $B \rightarrow X_{s} \gamma \frac{15}{10}$. The branching ratio $\left(B \rightarrow K^{*} \gamma\right)=(4.5 \pm 1.5 \pm 0.9) \times 10^{-5}$ was in agreement with the SM estimates, but theoretical uncertainty was large. Measuring the Inclusive process $B \rightarrow X_{s} \gamma$ was challenging, but as the photon energy spectrum in this process was already calculated in 1990 by Christoph Greub and me ${ }^{16}$, this came in handy for the CLEO measurements ${ }^{17}$ shown in Fig. 1 (left frame) and compared with the theoretical prediction 16 , Since then, a lot of experimental and theoretical effort has gone in the precise measurements and in performing higher order perturbative and nonperturbative calculations. As a consequence, $B \rightarrow X_{s} \gamma$ has now become the standard candle of FCNC processes, with the measured branching ratio and the precise higher order SM-based calculation providing valuable constraints on the parameters of BSM physics. The impact of the $B$-factories on this measurement can be judged by the scale in Fig. 1 (right frame), which is due to the Belle collaboration $\frac{18}{}$.
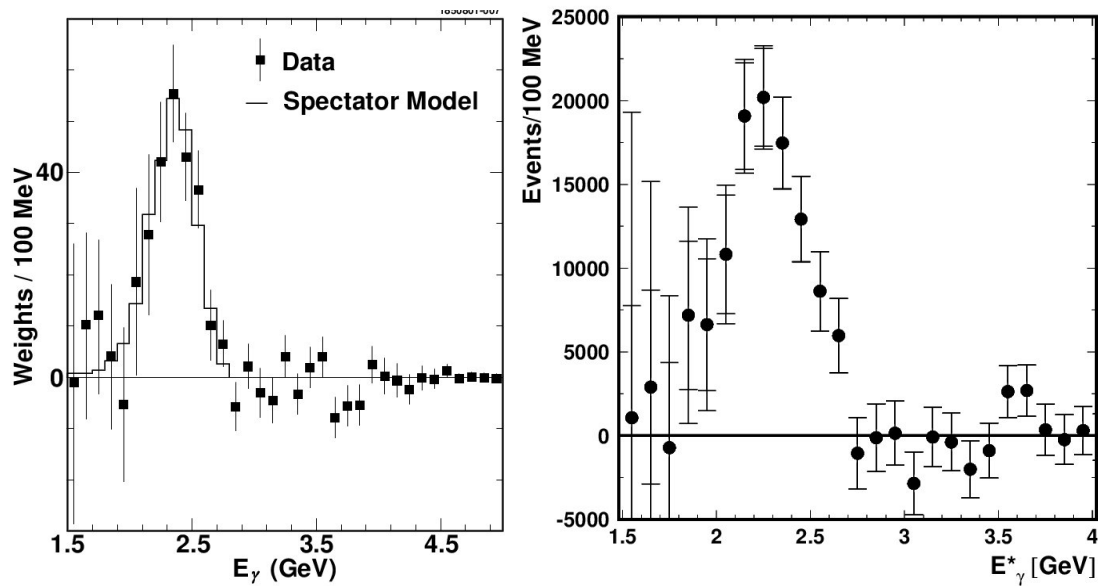

Fig. 1. Photon energy spectrum in the inclusive decay $B \rightarrow X_{s} \gamma$ measured by CLEO (left frame ${ }^{17}$ and Belle (right frame) 18 .

The next frontier of rare $B$-decays involves the so-called electroweak penguins, which govern the decays of the inclusive processes $B \rightarrow\left(X_{s}, X_{d}\right) \ell^{+} \ell^{-}$and the exclusive decays such as $B \rightarrow\left(K, K^{*}, \pi\right) \ell^{+} \ell^{-}$. These processes have rather small branching ratios and hence they were first measured at the $B$-factories. Inclusive decays remain their domain, but experiments at the LHC, in particular, LHCb, are now at the forefront of exclusive semileptonic decays. Apart from these, also the leptonic $B$-decays $B_{s} \rightarrow \mu^{+} \mu^{-}$and $B_{d} \rightarrow \mu^{+} \mu^{-}$have been measured at the LHC.

I will review some of the key measurements and the theory relevant for their 
interpretation. This description is anything but comprehensive, for which I refer to some recent excellent references $\frac{19}{22}$ and resources, such as HFAG ${ }^{23}$ and FLAG ${ }^{\sqrt{9}}$.

\subsection{Inclusive decays $B \rightarrow X_{s} \gamma$ at $N N L O$ in the $S M$}

The leading order diagrams for the decay $b \rightarrow s \gamma$ are shown are shown in Fig. 2 , including also the tree diagram for $b \rightarrow u \bar{u} s \gamma$, which yields a soft photon. The first two diagrams are anyway suppressed due to the CKM matrix elements, as indicated. The charm- and top- quark contributions enter with opposite signs, and the relative contributions indicted are after including the leading order (in $\alpha_{s}$ ) QCD effects. A typical diagram depicting perturbative QCD corrections due to the exchange of a gluon is also shown.
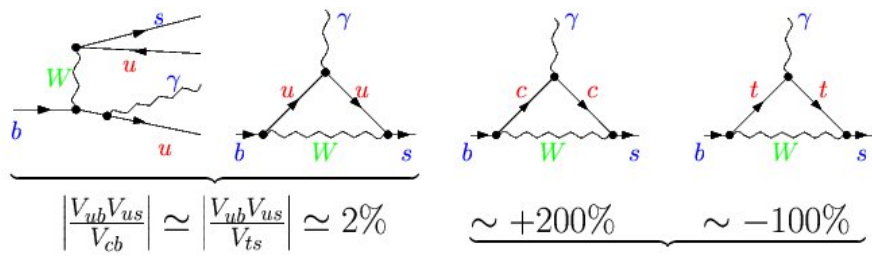

$$
\underbrace{\sim+200 \% \quad \sim-100 \%}
$$

In the amplitude, after including LO QCD effects.

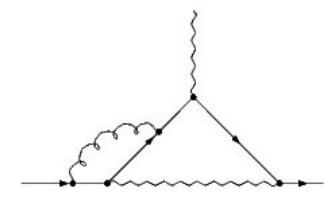

Fig. 2. Examples of the leading electroweak diagrams for $B \rightarrow X_{s} \gamma$ from the up, charm, and top quarks. A diagram involving a gluon exchange is shown in the lower figure.

The QCD logarithms $\alpha_{s} \ln M_{W}^{2} / m_{b}^{2}$ enhance the branching ratio $\mathcal{B}\left(B \rightarrow X_{s} \gamma\right)$ by more than a factor 2 , and hence such logs have to be resummed. This is done using an effective field theory approach, obtained by integrating out the top quark and the $W^{ \pm}$bosons. Keeping terms up to dimension-6, the effective Lagrangian for $B \rightarrow X_{s} \gamma$ and $B \rightarrow X_{s} \ell^{+} \ell^{-}$reads as follows:

$$
\mathcal{L}=\mathcal{L}_{Q C D \times Q E D}(q, l)+\frac{4 G_{F}}{\sqrt{2}} V_{t s}^{*} V_{t b} \sum_{i=1}^{10} C_{i}(\mu) O_{i}
$$




$$
\begin{aligned}
&(q=u, d, s, c, b, \quad l=e, \mu, \tau) \\
& O_{i}=\left\{\begin{array}{lll}
\left(\bar{s} \Gamma_{i} c\right)\left(\bar{c} \Gamma_{i}^{\prime} b\right), & i=1,(2), & C_{i}\left(m_{b}\right) \sim-0.26(1.02) \\
\left(\bar{s} \Gamma_{i} b\right) \Sigma_{q}\left(\bar{q} \Gamma_{i}^{\prime} q\right), & i=3,4,5,6, & \left|C_{i}\left(m_{b}\right)\right|<0.08 \\
\frac{e m_{b}}{16 \pi^{2}} \bar{s}_{L} \sigma^{\mu \nu} b_{R} F_{\mu \nu}, & i=7, & C_{7}\left(m_{b}\right) \sim-0.3 \\
\frac{g m_{b}}{16 \pi^{2}} \bar{s}_{L} \sigma^{\mu \nu} T^{a} b_{R} G_{\mu \nu}^{a}, & i=8, & C_{8}\left(m_{b}\right) \sim-0.16 \\
\frac{e^{2}}{16 \pi^{2}}\left(\bar{s}_{L} \gamma_{\mu} b_{L}\right)\left(\bar{l}^{\mu}\left(\gamma_{5}\right) l\right), i=9,(10) & C_{i}\left(m_{b}\right) \sim 4.27(-4.2)
\end{array}\right.
\end{aligned}
$$

Here, $G_{F}$ is the Fermi coupling constant, $V_{i j}$ are the CKM matrix elements, $O_{i}$ are the four-Fermi and dipole operators, and $C_{i}(\mu)$ are the Wilson coefficients, evaluated at the scale $\mu$, which is taken typically as $\mu=m_{b}$, and their values in the NNLO accuracy are given above for $\mu=4.8 \mathrm{GeV}$. Variations due to a different choice of $\mu$ and uncertainties from the upper scale-setting $m_{t} / 2 \leq \mu_{0} \leq 2 m_{t}$ can be seen elsewhere 19 .

There are three essential steps of the calculation:

- Matching: Evaluating $C_{i}\left(\mu_{0}\right)$ at $\mu_{0} \sim M_{W}$ by requiring the equality of the SM and the effective theory Green functions.

- Mixing: Deriving the effective theory renormalisation group equation $\overline{(\mathrm{RGE})}$ and evolving $C_{i}(\mu)$ from $\mu_{0}$ to $\mu_{b} \sim m_{b}$.

- Matrix elements: Evaluating the on-shell amplitudes at $\mu_{b} \sim m_{b}$.

All three steps have been improved in perturbation theory and now include the next-to-next-to-leading order effects (NLLO), i.e., contributions up to $O\left(\alpha_{s}^{2}\left(m_{b}\right)\right)$. A monumental theoretical effort stretched well over a decade with the participation of a large number of theorists underlies the current theoretical precision of the branching ratio. The result is usually quoted for a threshold photon energy to avoid experimental background from other Bremsstrahlung processes. For the decay with $E_{\gamma}>1.6 \mathrm{GeV}$ in the rest frame of the $B$ meson, the result at NNLO accuracy is $24 \sqrt{25}$

$$
\mathcal{B}\left(B \rightarrow X_{s} \gamma\right)=(3.36 \pm 0.23) \times 10^{-4},
$$

where the dominant SM uncertainty is non-perturbative 26 . This is to be compared with the current experimental average of the same ${ }^{23}$

$$
\mathcal{B}\left(B \rightarrow X_{s} \gamma\right)=(3.43 \pm 0.21 \pm 0.07) \times 10^{-4},
$$

where the first error is statistical and the second systematic, yielding a ratio $1.02 \pm$ 0.08 , providing a test of the SM to an accuracy better than $10 \%$.

The CKM-suppressed decay $B \rightarrow X_{d} \gamma$ has also been calculated in the NNLO precision. The result for $E_{\gamma}>1.6 \mathrm{GeV}$ is 24

$$
\mathcal{B}\left(B \rightarrow X_{d} \gamma\right)=\left(1.73_{-0.22}^{+0.12}\right) \times 10^{-5}
$$


This will be measured precisely at Belle II. The constraints on the CP asymmetry are not very restrictive, but the current measurements are in agreement with the SM expectation. For further details, see $\mathrm{HFAG}^{23}$.

\subsection{Bounds on the charged Higgs mass from $\mathcal{B}\left(B \rightarrow X_{s} \gamma\right)$}

As the agreement between the SM and data is excellent, the decay rate for $B \rightarrow X_{s} \gamma$ provides constraints on the parameters of the BSM theories, such as supersymmetry and the 2 Higgs-doublet models (2HDM). In calculating the BSM effects, depending on the model, the SM operator basis may have to be enlarged, but in many cases one anticipates additive contributions to the Wilson coefficients in the SM basis. In the context of $B \rightarrow X_{s} \gamma$, it is customary to encode the BSM effects in the Wilson coefficients of the dipole operators $C_{7}(\mu)$ and $C_{8}(\mu)$, and the constraints from the branching ratio on the additive coefficients $\Delta C_{7}$ and $\Delta C_{8}$ then takes the numerical form 24

$$
\mathcal{B}\left(B \rightarrow X_{s} \gamma\right) \times 10^{4}=(3.36 \pm 0.23)-8.22 \Delta C_{7}-1.99 \Delta C_{8}
$$

To sample the kind of constraints that can be derived on the parameters of the BSM models, the $2 \mathrm{HDM}$ is a good case, as the branching ratio for the decay $B \rightarrow X_{s} \gamma$ in this model has been derived to the same theoretical accuracy ${ }^{28}$. The Lagrangian for the $2 \mathrm{HDM}$ is

$$
\mathcal{L}_{H^{+}}=\left(2 \sqrt{2} G_{F}\right)^{1 / 2} \Sigma_{i, j=1}^{3} \bar{u}_{i}\left(A_{u} m_{u_{i}} V_{i j} P_{L}-A_{d} m_{d_{j}} V_{i j} P_{R}\right) d_{j} H^{*}+\text { h.c. },
$$

where $V_{i j}$ are the CKM matrix elements and $P_{L / R}=\left(1 \mp \gamma_{5}\right) / 2$. The $2 \mathrm{HDM}$ contributions to the Wilson coefficients are proportional to $A_{i} A_{j}^{*}$, representing the contributions from the up-type $A_{u}$ and down-type $A_{d}$ quarks. They are defined in terms of the ratio of the vacuum expectation values, called $\tan \beta$, and are model dependent.

- $2 \mathrm{HDM}$ of type-I: $A_{u}=A_{d}=\frac{1}{\tan \beta}$,

- $2 \mathrm{HDM}$ of type-II: $A_{u}=-1 / A_{d}=\frac{1}{\tan \beta}$.

Examples of Feynman diagrams contributing to $B \rightarrow X_{s} \gamma$ in the $2 \mathrm{HDM}$ are shown in Fig. 3. Apart from $\tan \beta$, the other parameter of the 2HDM is the mass of the charged Higgs $M_{H}^{ \pm}$. As $\mathcal{B}\left(B \rightarrow X_{s} \gamma\right)$ becomes insensitive to $\tan \beta$ for larger values, $\tan \beta>2$, the $2 \mathrm{HDM}$ contribution depends essentially on $M_{H}^{ \pm}$. The current measurements and the SM estimates then provide constraints on $M_{H}^{ \pm}$, as shown in Fig. 4. a updated using 2428 , yielding ${ }^{24} M_{H}^{ \pm}>480 \mathrm{GeV}$ ( $90 \%$ C.L.) and $M_{H}^{ \pm}>358$ $\mathrm{GeV}$ ( $99 \%$ C.L.) . These constraints are competitive to the direct searches of the $H^{ \pm}$at the LHC.

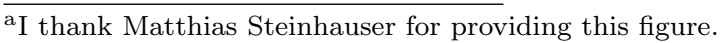



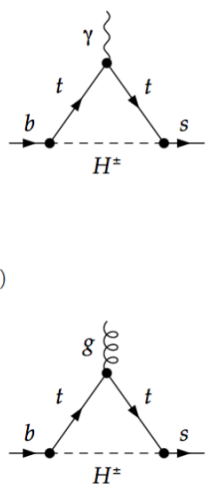

(b)

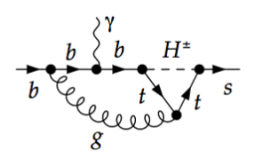

(e)

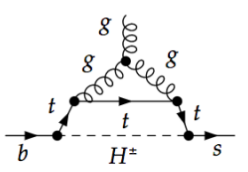

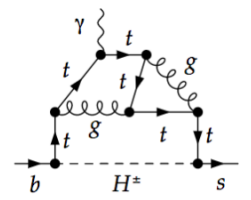

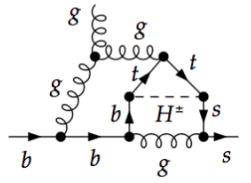

Fig. 3. Sample Feynman diagrams that matter for $B \rightarrow X_{s} \gamma$ in the $2 \mathrm{HDM}^{28}$. $H^{ \pm}$denotes a charged Higgs.

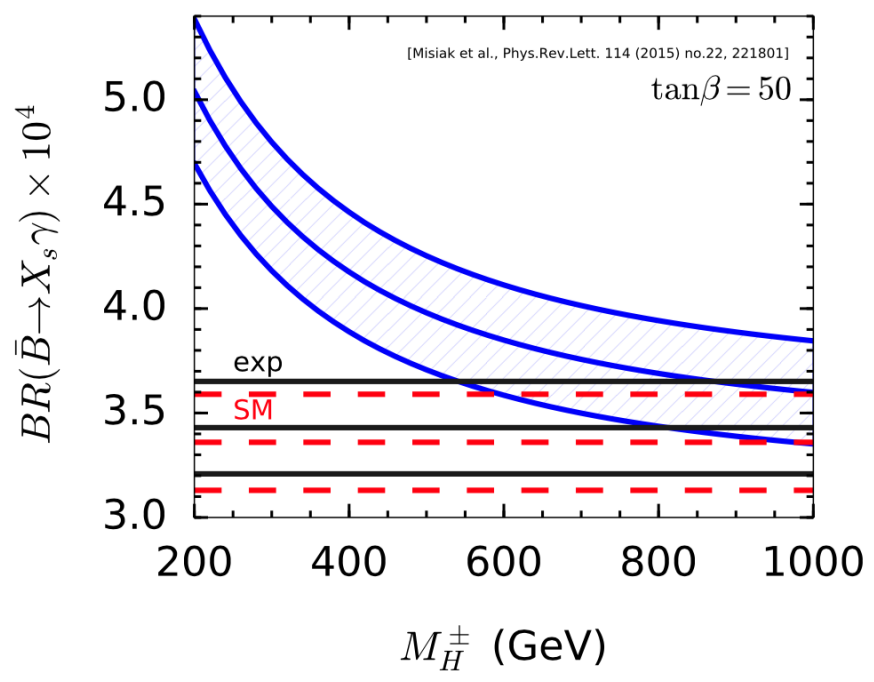

Fig. 4. Constraints on the charged Higgs mass $m_{H}^{ \pm}$from $\mathcal{B}\left(B \rightarrow X_{s} \gamma\right)$ in the $2 \mathrm{HDM}^{24 \mid 28}$. Measured branching ratio (exp) and the SM estimates are also shown. The curves demarcate the central values and $\pm 1 \sigma$ errors.

\subsection{Exclusive radiative rare $B$ decays}

Exclusive radiative decays, such as $B \rightarrow V \gamma\left(V=K^{*}, \rho, \omega\right)$ and $B_{s} \rightarrow \phi \gamma$, have been well-measured at the $B$ factories. In addition, they offer the possibility of measuring CP- and isospin asymmetries, a topic I will not discuss here. Theoretically, exclusive decays are more challenging, as they require the knowledge of the form factors at $q^{2}=0$, which can not be calculated directly using Lattice QCD. However, 
light-cone QCD sum rules ${ }^{12 \mid 13}$ also do a good job for calculating heavy $\rightarrow$ light form factors at low- $q^{2}$. In addition, the matrix elements require gluonic exchanges between the spectator quark and the active quarks (spectator-scattering), introducing intermediate scales in the decay rates. Also long-distance effects generated by the four-quark operators with charm quarks are present and are calculable in limited regions 29 . Thus, exclusive decays are theoretically not as precise as the inclusive decay $B \rightarrow X_{s} \gamma$. However, techniques embedded in HQET and SCET have led to the factorisation of the decay matrix elements into perturbatively calculable (hard) and non-perturbative (soft) parts, akin to the deep inelastic scattering processes. These factorisation-based approaches are the main work-horse in this field. Renormalisation group (RG) methods then allow to sum up large logarithms, and this program has been carried out to a high accuracy.

A detailed discussion of the various techniques requires a thorough review, which can't be carried out here. I will confine myself by pointing to some key references, beginning from the QCD factorisation approach, pioneered by Beneke, Buchalla, Neubert and Sachrajda ${ }^{30}$, which has been applied to the radiative decays $B \rightarrow\left(K^{*}, \rho, \omega\right) \gamma^{\underline{31} 34}$. Another theoretical framework, called pQCD ${ }^{35136}$, has also

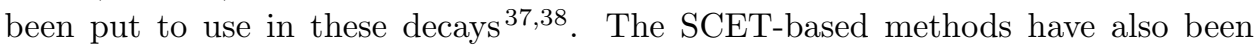
harnessed 39140 . The advantage of SCET is that it allows for an unambiguous separation of the scales, and an operator definition of each object in the factorisation formula can be given. Following the QCD factorisation approach, a factorisation formula for the $B \rightarrow V \gamma$ matrix element can be written in SCET as well

$$
\left\langle V \gamma\left|Q_{i}\right| B\right\rangle=\Delta_{i} C^{A} \xi_{V_{\perp}}+\frac{\sqrt{m_{B}} F f_{V_{\perp}}}{4} \int d w d u \phi_{+}^{B}(w) \phi_{\perp}^{V}(u) t_{i}^{I I},
$$

where $F$ and $f_{V_{\perp}}$ are meson decay constants; $\phi_{+}^{B}(w)$ and $\phi_{\perp}^{V}(u)$ are the light-cone distribution amplitudes for the $B$ - and $V$-meson, respectively. The SCET form factor $\xi_{V_{\perp}}$ is related to the QCD form factor through perturbative and power corrections, and the perturbative hard QCD kernels are the coefficients $\Delta_{i} C^{A}$ and $t_{i}^{I I}$. They are known to complete NLO accuracy in RG-improved perturbation theory 4.

The factorisation formula (6) has been calculated to NNLO accuracy in SCET 41 (except for the NNLO corrections from the spectator scattering). As far as the decays $B \rightarrow K^{*} \gamma$ and $B_{s} \rightarrow \phi \gamma$ are concerned, the partial NNLO theory is still the state-of-the-art. Their branching ratios as well as the ratio of the decay rates $\mathcal{B}\left(B_{s} \rightarrow \phi \gamma / \mathcal{B}\left(B \rightarrow K^{*} \gamma\right)\right.$ are given in Table 1, together with the current experimental averages 23 . The corresponding calculations for the CKM-suppressed decays $B \rightarrow(\rho, \omega) \gamma$ are not yet available to the desired theoretical accuracy, due to the annihilation contributions, for which, to the best of my knowledge, no factorisation theorem of the kind discussed above has been proven. The results from a QCD-Factorisation based approach ${ }^{32}$ for $B \rightarrow \rho \gamma$ are also given in Table 1 and compared with the data. The exclusive decay rates shown are in agreement with the experimental measurements, though theoretical precision is not better than $20 \%$. Obviously, there is need for a better theoretical description, more so as Belle II will 
measure the radiative decays with greatly improved precision. I will skip a discussion of the isospin and CP asymmetries in these decays, as the current experimental bounds $\frac{23}{23}$ are not yet probing the SM in these observables.

Table 1. Measurements [HFAG 2014] $]^{23}$ and SM-based estimates of $\mathcal{B}\left(B \rightarrow\left(K^{*}, \rho\right) \gamma\right)$ and $\mathcal{B}\left(B_{s} \rightarrow \phi \gamma\right)$ in units of $10^{-5}$, and the ratio $\mathcal{B}\left(B_{s} \rightarrow \phi \gamma\right) / \mathcal{B}\left(B^{0} \rightarrow K^{* 0} \gamma\right)$

\begin{tabular}{|l|l|l|}
\hline Decay Mode & Expt. (HFAG) & Theory (SM) \\
\hline$B^{0} \rightarrow K^{* 0} \gamma$ & $4.33 \pm 0.15$ & $4.6 \pm 1.4$ \\
\hline$B^{+} \rightarrow K^{*+} \gamma$ & $4.21 \pm 0.18$ & $4.3 \pm 1.4$ \\
\hline$B_{s} \rightarrow \phi \gamma$ & $3.59 \pm 0.36$ & $4.3 \pm 1.4$ \\
\hline$B_{s} \rightarrow \phi \gamma / B^{0} \rightarrow K^{* 0} \gamma$ & $0.81 \pm 0.08$ & $1.0 \pm 0.2$ \\
\hline$B^{0} \rightarrow \rho^{0} \gamma$ & $0.86_{-0.14}^{+0.15}$ & $0.65 \pm 0.12$ \\
\hline$B^{+} \rightarrow \rho^{+} \gamma$ & $0.98 \pm 0.25$ & $1.37 \pm 0.26$ \\
\hline \multicolumn{2}{|l}{} \\
\hline
\end{tabular}

\section{Semileptonic $b \rightarrow s$ decays $B \rightarrow\left(X_{s}, K, K^{*}\right) \ell^{+} \ell^{-}$}

There are two $b \rightarrow s$ semileptonic operators in SM:

$$
O_{i}=\frac{e^{2}}{16 \pi^{2}}\left(\bar{s}_{L} \gamma_{\mu} b_{L}\right)\left(\bar{l}^{\mu}\left(\gamma_{5}\right) l\right), \quad i=9,(10)
$$

Their Wilson Coefficients have the following perturbative expansion:

$$
\begin{aligned}
C_{9}(\mu) & =\frac{4 \pi}{\alpha_{s}(\mu)} C_{9}^{(-1)}(\mu)+C_{9}^{(0)}(\mu)+\frac{\alpha_{s}(\mu)}{4 \pi} C_{9}^{(1)}(\mu)+\ldots \\
C_{10} & =C_{10}^{(0)}+\frac{\alpha_{s}\left(M_{W}\right)}{4 \pi} C_{10}^{(1)}+\ldots
\end{aligned}
$$

The term $C_{9}^{(-1)}(\mu)$ reproduces the electroweak logarithm that originates from the photonic penguins with charm quark loops, shown below 42 .

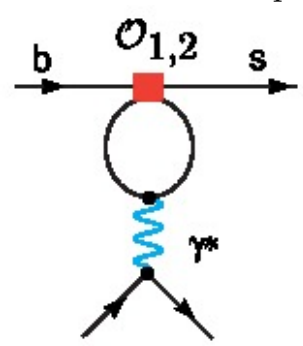


The first two terms in the perturbative expansion of $C_{9}\left(m_{b}\right)$ are

$$
C_{9}^{(0)}\left(m_{b}\right) \simeq 2.2 ; \quad \frac{4 \pi}{\alpha_{s}\left(m_{b}\right)} C_{9}^{(-1)}\left(m_{b}\right)=\frac{4}{9} \ln \frac{M_{W}^{2}}{m_{b}^{2}}+\mathcal{O}\left(\alpha_{s}\right) \simeq 2 .
$$

As they are very similar in magnitude, one needs to calculate the NNLO contribution to get reliable estimates of the decay rate. In addition, leading power corrections in $1 / m_{c}$ and $1 / m_{b}$ are required.

\subsection{Inclusive semileptonic decays $B \rightarrow X_{s} \ell^{+} \ell^{-}$}

A lot of theoretical effort has gone into calculating the perturbative QCD NNLO , electromagnetic logarithms and power corrections 42 46. The B-factory experiments Babar and Belle have measured the dilepton invariant mass spectrum $d \mathcal{B}(B \rightarrow$ $\left.X_{s} \ell^{+} \ell^{-}\right) / d q^{2}$ practically in the entire kinematic region and have also measured the so-called Forward-backward lepton asymmetry $A_{F B}\left(q^{2}\right)^{47}$. They are shown in Fig. 5 , and compared with the SM-based theoretical calculations. Note that a cut of $q^{2}>$ $0.2 \mathrm{GeV}^{2}$ on the dilepton invariant squared mass is used. As seen in these figures, two resonant regions near $q^{2}=M_{J / \psi}^{2}$ and $q^{2}=M_{J / \psi^{\prime}}^{2}$ have to be excluded when comparing with the short-distance contribution. They make up what is called the long-distance contribution from the processes $B \rightarrow X_{s}+\left(J / \psi, J / \psi^{\prime}\right) \rightarrow X_{s}+\ell^{+} \ell^{-}$, whose dynamics is determined by the hadronic matrix elements of the operators $O_{1}$ and $\mathrm{O}_{2}$. They have also been calculated via a dispersion relation $\underline{48}$ and data on the measured quantity $R_{\text {had }}(s)=\sigma\left(e^{+} e^{-} \rightarrow\right.$ hadrons $) / \sigma\left(e^{+} e^{-} \rightarrow \mu^{+} \mu^{-}\right)$, and in some analyses are also included. As the (short-distance) contribution is expected to be a smooth function of $q^{2}$, one uses the perturbative distributions in interpolating these regions as well. The experimental distributions are in agreement with the SM, including also the zero point of $A_{F B}\left(q^{2}\right)$, which is a sensitive function of the ratio of the two Wilson coefficients $C_{9}$ and $C_{10}$.

The branching ratio for the inclusive decay $B \rightarrow X_{s} \ell^{+} \ell^{-}$with a lower cut on the dilepton invariant mass $q^{2}>0.2 \mathrm{GeV}^{2}$ at NNLO accuracy is 44

$$
\mathcal{B}\left(B \rightarrow X_{s} \ell^{+} \ell^{-}\right)=(4.2 \pm 0.7) \times 10^{-6},
$$

to be compared with the current experimental average of the same $\mathrm{e}^{23}$

$$
\mathcal{B}\left(B \rightarrow X_{s} \ell^{+} \ell^{-}\right)=\left(3.66_{-0.77}^{+0.76}\right) \times 10^{-6} .
$$

The two agree within theoretical and experimental errors. The experimental cuts which are imposed to remove the $J / \psi$ and $\psi^{\prime}$ resonant regions are indicated in Fig. 5. The effect of logarithmic QED corrections becomes important for more restrictive cuts on $q^{2}$, and they have been worked out for different choices of the $q^{2}$-range in a recent paper $\underline{49}$.

\subsection{Exclusive Decays $B \rightarrow\left(K, K^{*}\right) \ell^{+} \ell^{-}$}

The $B \rightarrow K$ and $B \rightarrow K^{*}$ transitions involve the following weak currents:

$$
\Gamma_{\mu}^{1}=\bar{s} \gamma_{\mu}\left(1-\gamma_{5}\right) b, \quad \Gamma_{\mu}^{2}=\bar{s} \sigma_{\mu \nu} q^{\nu}\left(1+\gamma_{5}\right) b .
$$



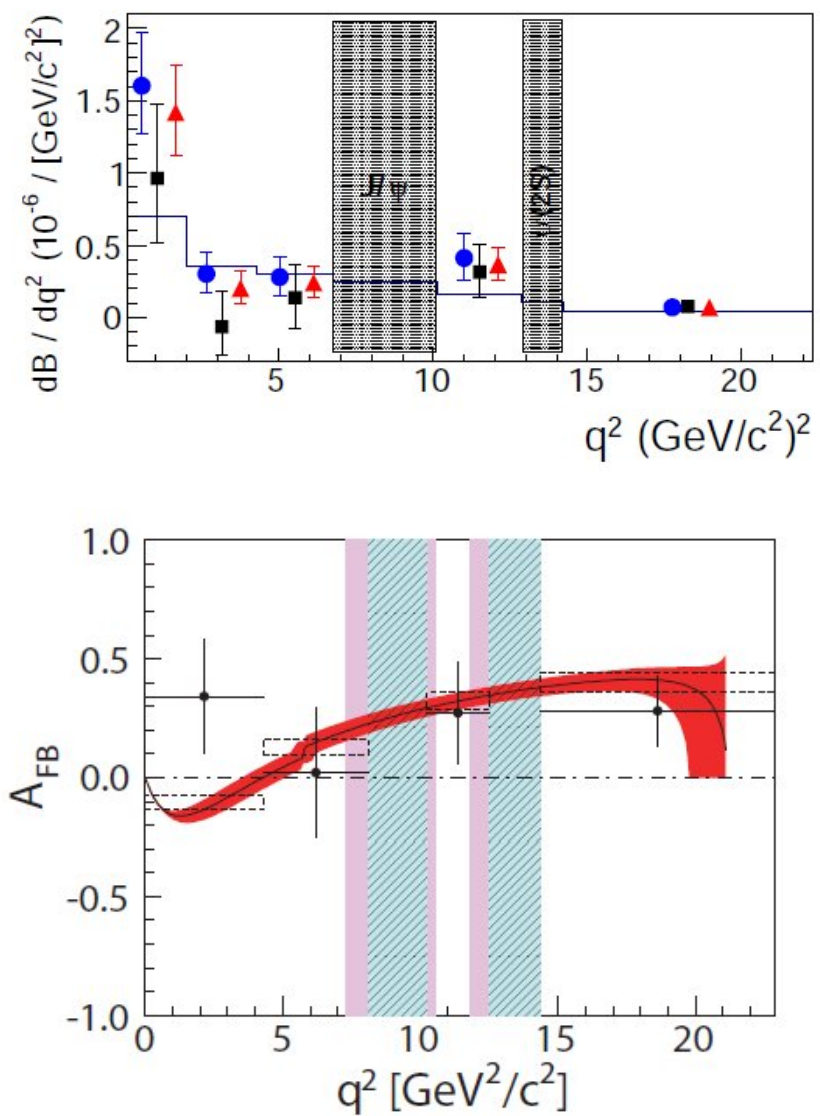

Fig. 5. Dilepton invariant mass Distribution measured by BaBar $\frac{50}{4}$ (upper frame) and the Forward-backward Asymmetry $A_{\mathrm{FB}}$ measured by Belle 51 (lower frame) in $B \rightarrow X_{s} \ell^{+} \ell^{-}$. The curve(above) and the band (below) are the SM expectations, discussed in the text.

Their matrix elements involve altogether 10 non-perturbative $q^{2}$-dependent functions (form factors), denoted by the following functions: ${ }^{\mathrm{b}}$

$$
\begin{aligned}
\left\langle K\left|\Gamma_{\mu}^{1}\right| B\right\rangle & \supset f_{+}^{K}\left(q^{2}\right), f_{-}^{K}\left(q^{2}\right) . \\
\left\langle K\left|\Gamma_{\mu}^{2}\right| B\right\rangle & \supset f_{T}^{K}\left(q^{2}\right) . \\
\left\langle K^{*}\left|\Gamma_{\mu}^{1}\right| B\right\rangle & \supset V\left(q^{2}\right), A_{1}\left(q^{2}\right), A_{2}\left(q^{2}\right), A_{3}\left(q^{2}\right) . \\
\left\langle K^{*}\left|\Gamma_{\mu}^{2}\right| B\right\rangle & \supset T_{1}\left(q^{2}\right), T_{2}\left(q^{2}\right), T_{3}\left(q^{2}\right) .
\end{aligned}
$$

Data on $B \rightarrow K^{*} \gamma$ provides normalisation of $T_{1}(0)=T_{2}(0) \simeq 0.28$. These form factors have been calculated using a number of non-perturbative techniques, in par-

\footnotetext{
${ }^{\mathrm{b}}$ As we will also discuss later the decays $B \rightarrow \pi \ell^{+} \ell^{-}$, we distinguish the $B \rightarrow K$ and $B \rightarrow \pi$ form factors by a superscript.
} 
ticular the QCD sum rules ${ }^{\sqrt{12152}}$ and Lattice $\mathrm{QCD}^{[53154156}$. They are complementary to each other, as the former are reliable in the low- $q^{2}$ domain and the latter can calculate only for large- $q^{2}$. They are usually combined to get reliable profiles of the form factors in the entire $q^{2}$ domain. However, heavy quark symmetry allows to reduce the number of independent form factors from 10 to 3 in low- $q^{2}$ domain $\left(q^{2} / m_{b}^{2} \ll 1\right)$. Symmetry-breaking corrections have been evaluated 57 . The decay rate, dilepton invariant mass distribution and the Forward-backward asymmetry in the low- $q^{2}$ region have been calculated for $B \rightarrow K^{*} \ell^{+} \ell^{-}$using the SCET formalism 58 . Current measurements of the branching ratios in the inclusive and exclusive semileptonic decays involving $b \rightarrow s$ transition are summarised in Table 2 and compared with the corresponding SM estimates. The inclusive measurements and the SM rates include a cut on the dilepton invariant mass $M_{\ell^{+} \ell^{-}}>0.2 \mathrm{GeV}$. They are in agreement with each other, though precision is currently limited due to the imprecise knowledge of the form factors.

\subsection{Current tests of lepton universality in semileptonic B-decays}

Currently, a number of measurements in $B$ decays suggests a breakdown of the lepton $(e, \mu, \tau)$ universality in semileptonic processes. In the SM, gauge bosons couple with equal strength to all three leptons and the couplings of the Higgs to a pair of charged lepton is proportional to the charged lepton mass, which are negligibly small for $\ell^{+} \ell^{-}=e^{+} e^{-}, \mu^{+} \mu^{-}$. Hence, if the lepton non-universality is experimentally established, it would be a fatal blow to the SM.

We briefly summarise the experimental situation starting from the decay $B^{ \pm} \rightarrow$ $K^{ \pm} \ell^{+} \ell^{-}$, whose decay rates were discussed earlier. Theoretical accuracy is vastly improved if instead of the absolute rates, ratios of the decay rates are computed. Data on the decays involving $K^{(*)} \tau^{+} \tau^{-}$is currently sparse, but first measurements of the ratios involving the final states $K^{(*)} \mu^{+} \mu^{-}$and $K^{(*)} e^{+} e^{-}$are available. In particular, a $2.6 \sigma$ deviation from the $e-\mu$ universality is reported by the $\mathrm{LHCb}$ collaboration in the ratio involving $B^{ \pm} \rightarrow K^{ \pm} \mu^{+} \mu^{-}$and $B^{ \pm} \rightarrow K^{ \pm} e^{+} e^{-}$measured in the low- $q^{2}$ region, which can be calculated rather accurately. In the interval $1 \leq q^{2} \leq 6 \mathrm{GeV}^{2}$, LHCb finds ${ }^{59}$

$$
R_{K} \equiv \frac{\Gamma\left(B^{ \pm} \rightarrow K^{ \pm} \mu^{+} \mu^{-}\right)}{\Gamma\left(B^{ \pm} \rightarrow K^{ \pm} e^{+} e^{-}\right)}=0.745_{-0.074}^{+0.090} \text { (stat) } \pm 0.035 \text { (syst) } .
$$

This ratio in the SM is close to 1 to a very high accuracy $\underline{60}$ over the entire $q^{2}$ region measured by the LHCb. Thus, the measurement in 100 amounts to about $2.6 \sigma$ deviation from the SM. Several BSM scenarios have been proposed to account for the $R_{K}$ anomaly, discussed below, including a $Z^{\prime}$-extension of the $\mathrm{SM}^{61}$. It should, however, be noted that the currently measured branching ratios $\mathcal{B}\left(B^{ \pm} \rightarrow K^{ \pm} e^{+} e^{-}\right)=$ $\left(1.56_{-0.15-0.4}^{+0.19+0.06}\right) \times 10^{-7}$ and $\mathcal{B}\left(B^{ \pm} \rightarrow K^{ \pm} \mu^{+} \mu^{-}\right)=(1.20 \pm 0.09 \pm 0.07) \times 10^{-7}$ are also lower than the SM estimates $\mathcal{B}^{\mathrm{SM}}\left(B^{ \pm} \rightarrow K^{ \pm} e^{+} e^{-}\right)=\mathcal{B}^{\mathrm{SM}}\left(B^{ \pm} \rightarrow K^{ \pm} \mu^{+} \mu^{-}\right)=$ $\left(1.75_{-0.29}^{+0.60}\right) \times 10^{-7}$, and the experimental error on the $\mathcal{B}\left(B^{ \pm} \rightarrow K^{ \pm} e^{+} e^{-}\right)$is twice as 
large. One has to also factor in that the electrons radiate very profusely (compared to the muons) and implementing the radiative corrections in hadronic machines is anything but straight forward. In coming years, this and similar ratios, which can also be calculated to high accuracy, will be measured with greatly improved precision at the LHC and Belle II.

The other place where lepton non-universality is reported is in the ratios of the decays $B \rightarrow D^{(*)} \tau \nu_{\tau}$ and $B \rightarrow D^{(*)} \ell \nu_{\ell}$. Defining

$$
R_{D^{(*)}}^{\tau / \ell} \equiv \frac{\mathcal{B}\left(B \rightarrow D^{(*)} \tau \nu_{\tau}\right) / \mathcal{B}^{\mathrm{SM}}\left(B \rightarrow D^{(*)} \tau \nu_{\tau}\right)}{\mathcal{B}\left(B \rightarrow D^{(*)} \ell \nu_{\ell}\right) / \mathcal{B}^{\mathrm{SM}}\left(B \rightarrow D^{(*)} \ell \nu_{\ell}\right)}
$$

the current averages of the BaBar, Belle, and the LHCb data $\operatorname{are}^{23}$ :

$$
R_{D}^{\tau / \ell}=1.37 \pm 0.17 ; \quad R_{D^{*}}^{\tau / \ell}=1.28 \pm 0.08
$$

This amounts to about $3.9 \sigma$ deviation from the $\tau / \ell(\ell=e, \mu)$ universality. Interestingly, this happens in a tree-level charged current process. If confirmed experimentally, this would call for a drastic contribution to an effective four-Fermi $L L$ operator $\left(\bar{c} \gamma_{\mu} b_{L}\right)\left(\tau_{L} \gamma_{\mu} \nu_{L}\right)$. It is then conceivable that the non-universality in $R_{K}$ (which is a loop-induced $b \rightarrow s$ process) ia also due to an $L L$ operator $\left(\bar{s} \gamma_{\mu} b_{L}\right)\left(\bar{\mu}_{L} \gamma_{\mu} \mu_{L}\right)$. Several suggestions along these lines involving a leptoquark have been made 62 . It is worth recalling that leptoquarks were introduced by Pati and Salam in 1973 in an attempt to unify leptons and quarks in $S U(4)^{65 \mid 66}$. The lepton non-universality in $B$ decays has revived the interest in theories with low-mass leptoquarks, discussed recently in a comprehensive work on this topic $\underline{67}$.

Table 2. Measurements [PDG 2014] and SM-based estimates 44 of the branching ratios $\mathcal{B}\left(B \rightarrow\left(X_{s}, K, K^{*}\right) \ell^{+} \ell^{-}\right)$in units of $10^{-6}$

\begin{tabular}{|l|l|l|}
\hline Decay Mode & Expt. (BELLE \& BABAR) & Theory (SM) \\
\hline$B \rightarrow K \ell^{+} \ell^{-}$ & $0.48 \pm 0.04$ & $0.35 \pm 0.12$ \\
\hline$B \rightarrow K^{*} e^{+} e^{-}$ & $1.19 \pm 0.20$ & $1.58 \pm 0.49$ \\
\hline$B \rightarrow K^{*} \mu^{+} \mu^{-}$ & $1.06 \pm 0.09$ & $1.19 \pm 0.39$ \\
\hline$B \rightarrow X_{s} \mu^{+} \mu^{-}$ & $4.3 \pm 1.2$ & $4.2 \pm 0.7$ \\
\hline$B \rightarrow X_{s} e^{+} e^{-}$ & $4.7 \pm 1.3$ & $4.2 \pm 0.7$ \\
\hline$B \rightarrow X_{s} \ell^{+} \ell^{-}$ & $4.5 \pm 1.0$ & $4.2 \pm 0.7$ \\
\hline & & \\
\hline
\end{tabular}




\subsection{Angular analysis of the decay $B^{0} \rightarrow K^{* 0}\left(\rightarrow K^{+} \pi^{-}\right) \mu^{+} \mu^{-}$}

For the inclusive decays $B \rightarrow X_{s} \ell^{+} \ell^{-}$, the observables which have been measured are the integrated rates, the dilepton invariant mass $d \Gamma / d q^{2}$ and the $\mathrm{FB}$ asymmetry $A_{\mathrm{FB}}\left(q^{2}\right)$. They are all found to be in agreement with the SM. In the exclusive decays such as $B \rightarrow K^{*} \ell^{+} \ell^{-}$and $B_{s} \rightarrow \phi \ell^{+} \ell^{-}$, a complete angular analysis of the decay is experimentally feasible. This allows one to measure a number of additional observables, defined below.

$$
\begin{aligned}
\frac{1}{d(\Gamma+\bar{\Gamma})} \frac{d^{4}(\Gamma+\bar{\Gamma})}{d q^{2} d \Omega}= & \frac{9}{32 \pi}\left[\frac{3}{4}\left(1-F_{L}\right) \sin ^{2} \theta_{K}+F_{L} \cos ^{2} \theta_{K}\right. \\
& +\frac{1}{4}\left(1-F_{L}\right) \sin ^{2} \theta_{K} \cos 2 \theta_{\ell}-F_{L} \cos ^{2} \theta_{K} \cos 2 \theta_{\ell} \\
& +S_{3} \sin ^{2} \theta_{K} \sin ^{2} \theta_{\ell} \cos 2 \phi+S_{4} \sin 2 \theta_{K} \sin 2 \theta_{\ell} \cos \phi \\
& +S_{5} \sin 2 \theta_{K} \sin \theta_{\ell} \cos \phi+\frac{4}{3} A_{\mathrm{FB}} \sin ^{2} \theta_{K} \cos \theta_{\ell} \\
& +S_{7} \sin 2 \theta_{K} \sin \theta_{\ell} \sin \phi+S_{8} \sin 2 \theta_{K} \sin 2 \theta_{\ell} \sin \phi \\
& \left.+S_{9} \sin ^{2} \theta_{K} \sin ^{2} \theta_{\ell} \sin 2 \phi\right] .
\end{aligned}
$$

The three angles $\theta_{K}, \theta_{\ell}$ and $\phi$ for the decay $B^{0} \rightarrow K^{* 0}\left(\rightarrow K^{+} \pi^{-}\right) \mu^{+} \mu^{-}$are defined in Fig. 6. An angular analysis of the decay chains $B^{0} \rightarrow K^{* 0}\left(\rightarrow K^{+} \pi^{-}\right) \mu^{+} \mu^{-68}$

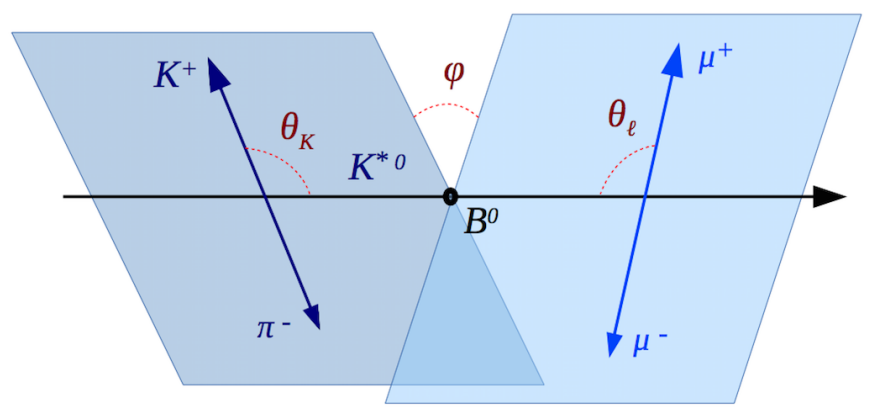

Fig. 6. Definitions of the angles in $B^{0} \rightarrow K^{* 0}\left(\rightarrow K^{+} \pi^{-}\right) \mu^{+} \mu^{-}$.

and $B_{s}^{0} \rightarrow \phi\left(\rightarrow K^{+} K^{-}\right) \mu^{+} \mu^{-69}$ has been carried out by LHCb.

The observables in $\sqrt{13}$ are $q^{2}$-dependent coefficients of the Wilson coefficients and hence they probe the underlying dynamics. Since these coefficients have been calculated to a high accuracy, the remaining theoretical uncertainty lies in the form factors and also from the charm-quark loops. The form factors have been calculated using the QCD sum rules and in the high- $q^{2}$ region also using lattice QCD. They limit the current theoretical accuracy. However, a number of so-called optimised observables has been proposed 70 , which reduce the dependence on the form factors. 
Using the $\mathrm{LHCb}$ convention, these observables are defined as 68

$$
\begin{aligned}
P_{1} \equiv & 2 S_{3} /\left(1-F_{L}\right) ; \quad P_{2} \equiv 2 A_{\mathrm{FB}} / 3\left(1-F_{L}\right) ; \quad P_{3} \equiv-S_{9} /\left(1-F_{L}\right), \\
& P_{4,5,6,8}^{\prime} \equiv S_{4,5,7,8} / \sqrt{F_{l}\left(1-F_{L}\right)} .
\end{aligned}
$$

These angular observables have been analysed in a number of theoretical stud-
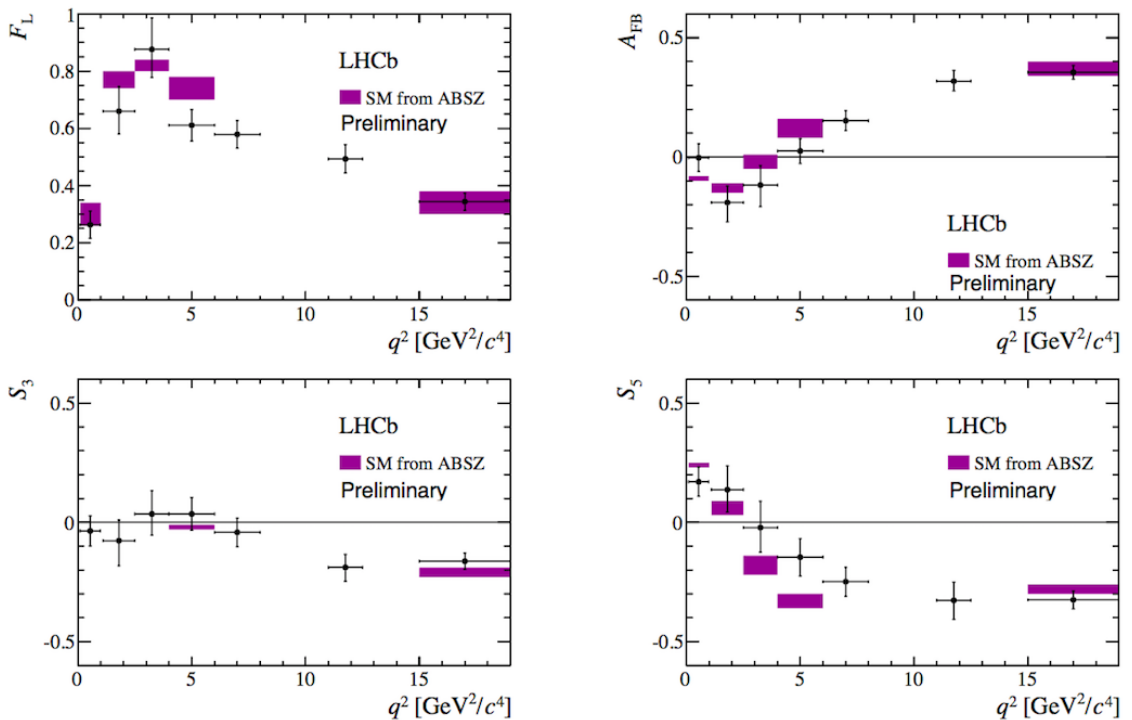

Fig. 7. CP-averaged variables in bins of $q^{2}$ for the observables $F_{\mathrm{L}}, A_{\mathrm{FB}}, S_{3}$ and $S_{5}$ in $B^{0} \rightarrow$ $K^{* 0}\left(\rightarrow K^{+} \pi^{-}\right) \mu^{+} \mu^{-}$measured by $\mathrm{LHCb}^{68}$ and comparison with the $\mathrm{SM}[73$.

ies $13 / 71 / 75$, which differ in the treatment of their non-perturbative input, mainly form factors. The $\mathrm{LHCb}$ collaboration, which currently dominates this field, has used these SM-based estimates and compared with their data in various $q^{2}$ bins. Two representative comparisons based on the theoretical estimates from Altmannshofer and Straub ${ }^{73}$ and Descotes-Genon, Hofer, Matias and Virto ${ }^{75}$ are shown in Figs. 7 and 8 , respectively. They are largely in agreement with the data, except for the distributions in the observables $S_{5}\left(q^{2}\right)$ (in Fig. 7) and $P_{5}^{\prime}\left(q^{2}\right)$ (in Fig. 8 in the bins around $q^{2} \geq 5 \mathrm{GeV}^{2}$. The pull on the SM depends on the theoretical model, reaching $3.4 \sigma$ in the bin $4.3 \leq q^{2} \leq 8.68 \mathrm{GeV}^{2}$ compared to DHMV 75 . There are deviations of a similar nature, between 2 and $3 \sigma$, seen in the comparison of $S_{5}$ and other quantities, such as the partial branching ratios in $B \rightarrow K^{*} \mu^{+} \mu^{-}$, $B_{s} \rightarrow \phi \mu^{+} \mu^{-}$and $F_{L}\left(q^{2}\right)^{73}$.

An analysis of the current Belle data ${ }^{76}$, shown in Fig. 9, displays a similar pattern as the one reported by LHCb. As the Belle data has larger errors, due to limited statistics, the resulting pull on the SM is less significant. In the interval $4.0 \leq q^{2} \leq 8.0 \mathrm{GeV}^{2}$, Belle reports deviations of $2.3 \sigma$ (compared to DHMV ${ }^{75}$ ), 

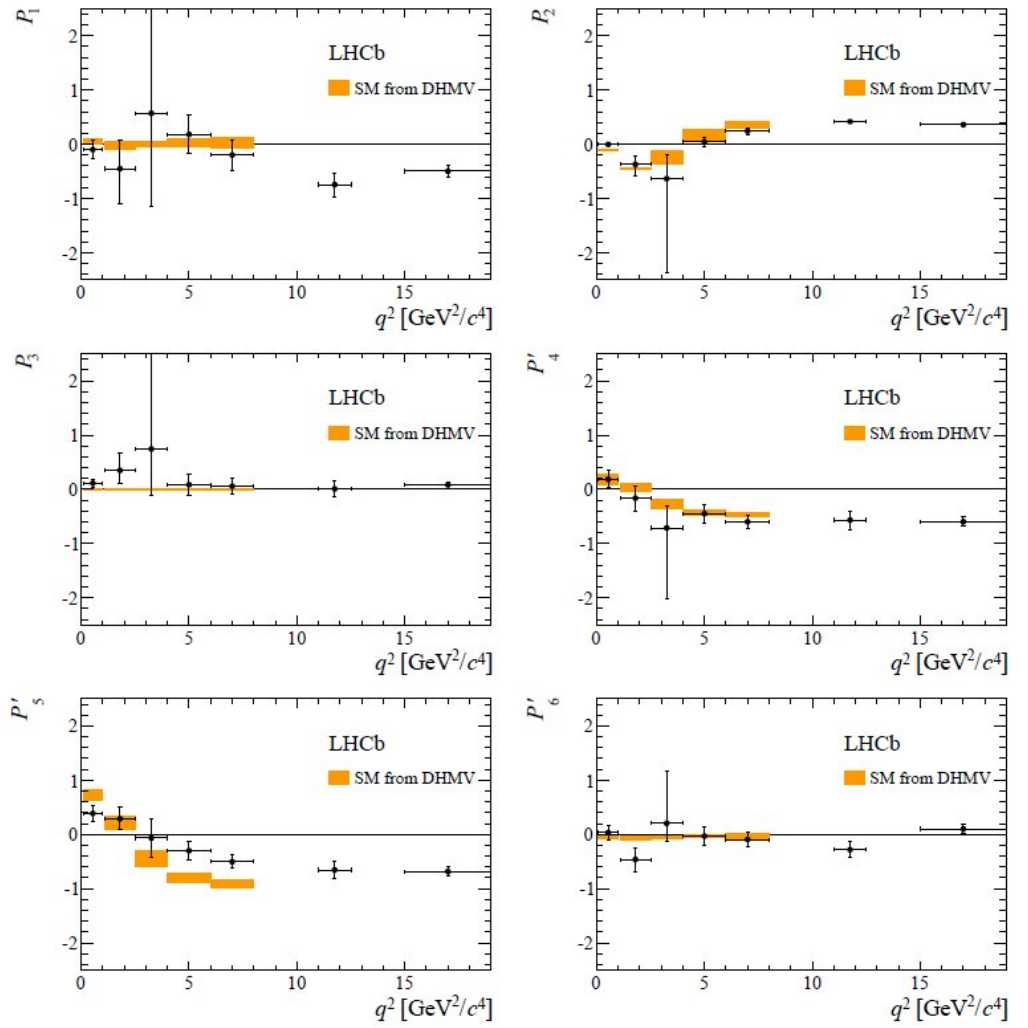

Fig. 8. The optimised angular observables in bins of $q^{2}$ in the decay $B^{0} \rightarrow K^{* 0}\left(\rightarrow K^{+} \pi^{-}\right) \mu^{+} \mu^{-}$ measured by the LHCb collaboration ${ }^{68}$ and comparison with the SM ${ }^{[75]}$.

$1.72 \sigma$ (compared to $\mathrm{BSZ}{ }^{13}$ ) and $1.68 \sigma$ (compared to $\mathrm{JC}^{72}$ ). These measurements will improve greatly at Belle II.

To quantify the deviation of the $\mathrm{LHCb}$ data from the SM estimates, a $\Delta \chi^{2}$ distribution for the real part of the Wilson coefficient $\operatorname{Re} C_{9}\left(m_{b}\right)$ is shown in Fig. 10. In calculating the $\Delta \chi^{2}$, the other Wilson coefficients are set to their SM values. The coefficient $\operatorname{Re} C_{9}^{\mathrm{SM}}\left(m_{b}\right)=4.27$ at the NNLO accuracy in the SM is indicated by a vertical line. The best fit of the $\mathrm{LHCb}$ data yields a value which is shifted from the SM, and the deviation in this coefficient is found to be $\Delta \operatorname{Re} C_{9}\left(m_{b}\right)=$ $-1.04 \pm 0.25$. The deviation is tantalising, but not yet conclusive. A bit of caution is needed here as the SM estimates used in the analysis above may have to be revised, once the residual uncertainties are better constrained. In particular, the hadronic contributions generated by the four-quark operators with charm are difficult to estimate, especially around $q^{2} \sim 4 m_{c}^{2}$, leading to an effective shift in the value of the Wilson coefficient being discussed 77 . 


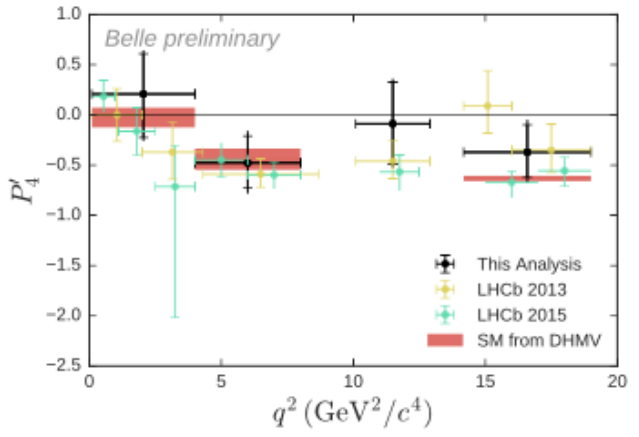

(a)Result for $P_{4}^{\prime}$

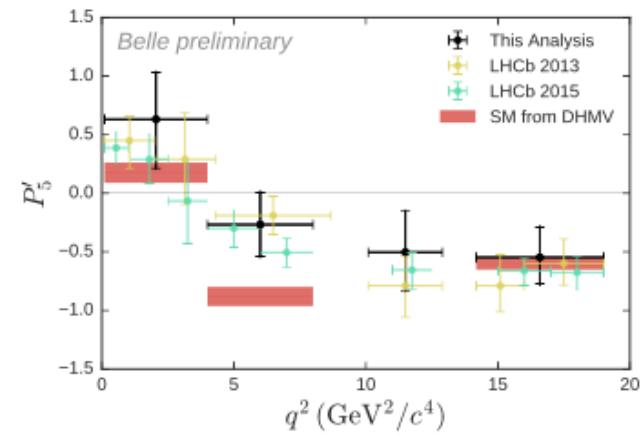

(b)Result for $P_{5}^{\prime}$

Fig. 9. The optimised angular observables $P_{4}^{\prime}$ and $P_{5}^{\prime}$ in bins of $q^{2}$ in the decay $B^{0} \rightarrow K^{* 0}(\rightarrow$ $\left.K^{+} \pi^{-}\right) \mu^{+} \mu^{-}$measured by Belle $\frac{76}{76}$ and comparison with the $\mathrm{SM}^{75}$.

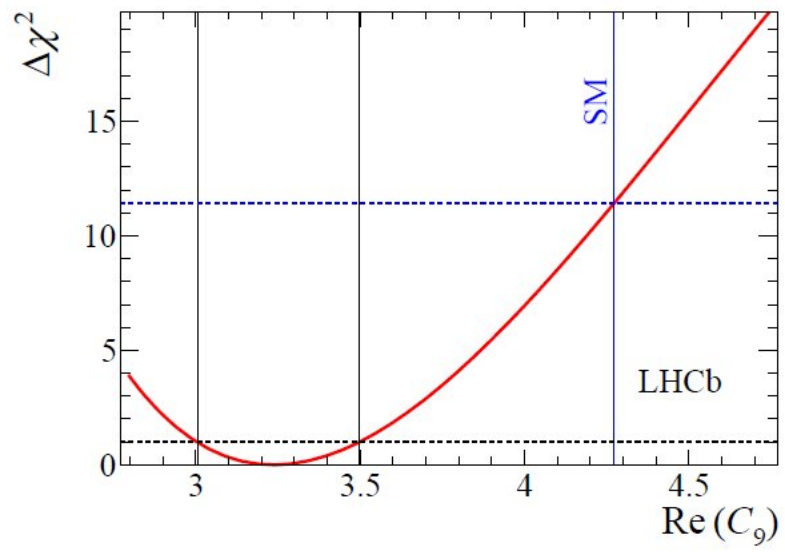

Fig. 10. The $\Delta \chi^{2}$ distribution for the real part of the Wilson coefficient $\operatorname{Re} C_{9}\left(m_{b}\right)$ from a fit of the CP-averaged observables $F_{\mathrm{L}}, A_{\mathrm{FB}}, S_{3}, \ldots, S_{9}$ in $B^{0} \rightarrow K^{* 0}\left(\rightarrow K^{+} \pi^{-}\right) \mu^{+} \mu^{-}$by the $\mathrm{LHCb}$ collaboration 68

\section{CKM-suppressed $b \rightarrow d \ell^{+} \ell^{-}$transitions in the SM}

Weak transitions $b \rightarrow d \ell^{+} \ell^{-}$, like the radiative decays $b \rightarrow d \gamma$, are CKM suppressed and because of this the structure of the effective weak Hamiltonian is different than the one encountered earlier for the $b \rightarrow s \ell^{+} \ell^{-}$transitions.

$$
\begin{aligned}
\mathcal{H}_{\mathrm{eff}}^{b \rightarrow d}= & -\frac{4 G_{G}}{\sqrt{2}}\left[V _ { t b } ^ { * } V _ { t d } \sum _ { i = 1 } ^ { 1 0 } C _ { i } ( \mu ) \left(O_{i}(\mu)\right.\right. \\
& \left.+V_{u b}^{*} V_{u d} \sum_{i=1}^{2} C_{i}(\mu)\left(O_{i}(\mu)-O_{i}(\mu)\right)\right]+ \text { h.c.. }
\end{aligned}
$$


Here $O_{i}(\mu)$ are the dimension-six operators introduced earlier (except for the interchange $s \rightarrow d$ quark) in $\mathcal{H}_{\text {eff }}^{b \rightarrow s}$. As the two CKM factors are comparable in magnitude $\left|V_{t b}^{*} V_{t d}\right| \simeq\left|V_{u b}^{*} V_{u d}\right|$, and have different weak phases, we anticipate sizeable $\mathrm{CP}$-violating asymmetries in both the inclusive $b \rightarrow d \ell^{+} \ell^{-}$and exclusive transitions, such as $B \rightarrow(\pi, \rho) \ell^{+} \ell^{-}$. The relevant operators appearing in $\mathcal{H}_{\mathrm{eff}}^{b \rightarrow d}$ are:

Tree operators

$$
\begin{gathered}
\mathcal{O}_{1}=\left(\bar{d}_{L} \gamma_{\mu} T^{A} c_{L}\right)\left(\bar{c}_{L} \gamma^{\mu} T^{A} b_{L}\right), \quad \mathcal{O}_{2}=\left(\bar{d}_{L} \gamma_{\mu} c_{L}\right)\left(\bar{c}_{L} \gamma^{\mu} b_{L}\right), \\
\mathcal{O}_{1}^{(u)}=\left(\bar{d}_{L} \gamma_{\mu} T^{A} u_{L}\right)\left(\bar{u}_{L} \gamma^{\mu} T^{A} b_{L}\right), \quad \mathcal{O}_{2}^{(u)}=\left(\bar{d}_{L} \gamma_{\mu} u_{L}\right)\left(\bar{u}_{L} \gamma^{\mu} b_{L}\right) .
\end{gathered}
$$

Dipole operators

$$
\mathcal{O}_{7}=\frac{e m_{b}}{g_{\mathrm{s}}^{2}}\left(\bar{d}_{L} \sigma^{\mu \nu} b_{R}\right) F_{\mu \nu}, \quad \mathcal{O}_{8}=\frac{m_{b}}{g_{\mathrm{s}}}\left(\bar{d}_{L} \sigma^{\mu \nu} T^{A} b_{R}\right) G_{\mu \nu}^{A}
$$

Semileptonic operators

$$
\mathcal{O}_{9}=\frac{e^{2}}{g_{\mathrm{s}}^{2}}\left(\bar{d}_{L} \gamma^{\mu} b_{L}\right) \sum_{\ell}\left(\bar{\ell} \gamma_{\mu} \ell\right), \quad \mathcal{O}_{10}=\frac{e^{2}}{g_{\mathrm{s}}^{2}}\left(\bar{d}_{L} \gamma^{\mu} b_{L}\right) \sum_{\ell}\left(\bar{\ell} \gamma_{\mu} \gamma_{5} \ell\right)
$$

Here, $e\left(g_{s}\right)$ is the QED (QCD) coupling constant. Since the inclusive decay $B \rightarrow$ $X_{d} \ell^{+} \ell^{-}$has not yet been measured, but hopefully will be at Belle II, we discuss the exclusive decay $B^{+} \rightarrow \pi^{+} \ell^{+} \ell^{-}$, which is the only $b \rightarrow d$ semileptonic transition measured so far.

\subsection{Exclusive decay $B^{+} \rightarrow \pi^{+} \ell^{+} \ell^{-}$}

The decay ${ }^{\mathrm{C}} B^{+} \rightarrow \pi^{+} \ell^{+} \ell^{-}$is induced by the vector and tensor currents and their matrix elements are defined as

$$
\begin{gathered}
\left\langle\pi\left(p_{\pi}\right)\left|\bar{b} \gamma^{\mu} d\right| B\left(p_{B}\right)\right\rangle=f_{+}^{\pi}\left(q^{2}\right)\left(p_{B}^{\mu}+p_{\pi}^{\mu}\right)+\left[f_{0}^{\pi}\left(q^{2}\right)-f_{+}^{\pi}\left(q^{2}\right)\right] \frac{m_{B}^{2}-m_{\pi}^{2}}{q^{2}} q^{\mu}, \\
\left\langle\pi\left(p_{\pi}\right)\left|\bar{b} \sigma^{\mu \nu} q_{\nu} d\right| B\left(p_{B}\right)\right\rangle=\frac{i f_{T}^{\pi}\left(q^{2}\right)}{m_{B}+m_{\pi}}\left[\left(p_{B}^{\mu}+p_{\pi}^{\mu}\right) q^{2}-q^{\mu}\left(m_{B}^{2}-m_{\pi}^{2}\right)\right] .
\end{gathered}
$$

These form factors are related to the ones in the decay $B \rightarrow K \ell^{+} \ell^{-}$, called $f_{i}^{K}\left(q^{2}\right)$, discussed earlier, by $S U(3)_{F}$ symmetry. Of these, the form factors $f_{+}^{\pi}\left(q^{2}\right)$ and $f_{0}^{\pi}\left(q^{2}\right)$ are related by isospin symmetry to the corresponding ones measured in the charged current process $B^{0} \rightarrow \pi^{-} \ell^{+} \nu_{\ell}$ by Babar and Belle, and they can be extracted from the data. This has been done using several parameterisations of the form factors with all of them giving an adequate description of the data ${ }^{78}$. Due to their analytic properties, the so-called z-expansion methods, in which the form factors are expanded in a Taylor series in $z$, employed in the Boyd-Grinstein-Lebed

${ }^{\mathrm{c}}$ Charge conjugation is implied here. 
(BGL) parametrisation ${ }^{79}$ and the Bourrely-Caprini-Lellouch (BCL) $)^{80}$ parametrisation, are preferable.

The BGL parametrisation is used in working out the decay rate and the invariant dilepton mass distribution ${ }^{78}$ for $B^{+} \rightarrow \pi^{+} \ell^{+} \ell^{-}$, which is discussed below. The BCL-parametrisation is used by the lattice-QCD groups, the HPQCD ${ }^{54 / 55}$ and Fermilab/MILC ${ }^{56}$ collaborations, to determine the form factors $f_{i}^{\pi}\left(q^{2}\right)$ and $f_{i}^{K}\left(q^{2}\right)$. In particular, the Fermilab/MILC collaboration has worked out the dilepton invariant mass distribution in the decay of interest $B^{+} \rightarrow \pi^{+} \ell^{+} \ell^{-}$, making use of their simulation in the large- $q^{2}$ region and extrapolating with the BCL parametrisation.

We first discuss the low- $q^{2}$ region $\left(q^{2} \ll m_{b}^{2}\right)$. In this case, heavy quark symmetry (HQS) relates all three form factors of interest $f_{i}^{\pi}\left(q^{2}\right)$ and this can be used advantageously to have a reliable estimate of the dilepton invariant mass spectrum in this region. Including lowest order HQS-breaking, the resulting expressions for the form factors (for $q^{2} / m_{b}^{2} \ll 1$ ) are worked out by Beneke and Feldmann $\frac{57}{}$. Thus, fitting the form factor $f_{+}\left(q^{2}\right)$ from the charged current data on $B \rightarrow \pi \ell^{+} \nu_{\ell}$ decay, and taking into account the HQS and its breaking, lead to a model-independent predictions of the differential branching ratio (dimuon mass spectrum) in the neutral current process $B^{+} \rightarrow \pi^{+} \ell^{+} \ell^{-}$for low- $q^{2}$ values. However, the long-distance contribution, which arises from the processes $B^{+} \rightarrow \pi^{+}\left(\rho^{0}, \omega\right) \rightarrow \pi^{+} \mu^{+} \mu^{-}$are not included here. The SM invariant dilepton mass distribution in $B^{+} \rightarrow \pi^{+} \ell^{+} \ell^{-}$ integrated over the range $1 \mathrm{GeV}^{2} \leq q^{2} \leq 8 \mathrm{GeV}^{2}$ yields a partial branching ratio

$$
\mathcal{B}\left(B^{+} \rightarrow \pi^{+} \mu^{+} \mu^{-}\right)=\left(0.57_{-0.05}^{+0.07}\right) \times 10^{-8} .
$$

Thanks to the available data on the charged current process and heavy quark symmetry, this enables an accuracy of about $10 \%$ for an exclusive branching ratio, comparable to the theoretical accuracy in the inclusive decay $B \rightarrow X_{s} \gamma$, discussed earlier. Thus, the decay $B^{+} \rightarrow \pi^{+} \mu^{+} \mu^{-}$offers a key advantage compared to the decay $B^{+} \rightarrow K^{+} \ell^{+} \ell^{-}$, in which case the charged current process is not available.

The differential branching ratio in the entire $q^{2}$ region is given by

$$
\frac{d \mathcal{B}\left(B^{+} \rightarrow \pi^{+} \ell^{+} \ell^{-}\right)}{d q^{2}}=C_{B}\left|V_{t b} V_{t d}^{*}\right|^{2} \sqrt{\lambda\left(q^{2}\right)} \sqrt{1-\frac{4 m_{\ell}^{2}}{q^{2}}} F\left(q^{2}\right)
$$

where the constant $C_{B}=G_{F}^{2} \alpha_{\mathrm{em}}^{2} \tau_{B} / 1024 \pi^{5} m_{B}^{3}$ and $\lambda\left(q^{2}\right)$ is the usual kinematic function $\lambda\left(q^{2}\right)=\left(m_{B}^{2}+m_{\pi}^{2}-q^{2}\right)^{2}-4 m_{B}^{2} m_{\pi}^{2}$. The function $F\left(q^{2}\right)$ depends on the effective Wilson coefficients, $C_{7}^{\text {eff }}, C_{9}^{\text {eff }}$, and $C_{10}^{\text {eff }}$, and the three form factors $f_{+}^{\pi}\left(q^{2}\right)$, $f_{0}^{\pi}\left(q^{2}\right)$ and $f_{T}^{\pi}\left(q^{2}\right)$. A detailed discussion of the determination of the form factors, of which only $f_{+}^{\pi}\left(q^{2}\right)$ and $f_{T}^{\pi}\left(q^{2}\right)$ are numerically important for $\ell^{ \pm}=e^{ \pm}, \mu^{ \pm}$is given elsewhere ${ }^{78}$. We recall that $f_{+}^{\pi}\left(q^{2}\right)$ is constrained by the data on the charged current process in the entire $q^{2}$ domain. In addition, the lattice-QCD results on the form factors in the large- $q^{2}$ domain and the HQS-based relations in the low- $q^{2}$ region provide sufficient constraints on the form factor. This has enabled a rather precise determination of the invariant dilepton mass distribution in $B^{+} \rightarrow \pi^{+} \ell^{+} \ell^{-}$. 
Taking into account the various parametric and form-factor dependent uncertainties, this yields the following estimate for the branching ratio for $B^{+} \rightarrow$ $\pi^{+} \mu^{+} \mu^{-82}$

$$
\mathcal{B}_{\mathrm{SM}}\left(B^{+} \rightarrow \pi^{+} \mu^{+} \mu^{-}\right)=\left(1.88_{-0.21}^{+0.32}\right) \times 10^{-8},
$$

to be compared with the measured branching ratio by the LHCb collaboration $\underline{82}$ (based on $3 \mathrm{fb}^{-1}$ data):

$$
\mathcal{B}_{\mathrm{LHCb}}\left(B^{+} \rightarrow \pi^{+} \mu^{+} \mu^{-}\right)=(1.83 \pm 0.24 \pm 0.05) \times 10^{-8},
$$

where the first error is statistical and the second systematic, resulting in excellent agreement. The dimuon invariant mass distribution measured by the $\mathrm{LHCb}$ collaboration $^{82}$ is shown in Fig. 11, and compared with the SM-based theoretical prediction, called APR13 $3^{78}$, and the lattice-based calculation, called FNAL/MILC 1556. Also shown is a comparison with a calculation, called $\mathrm{HKR}^{81}$, which has essentially the same short-distance contribution in the low- $q^{2}$ region, as discussed earlier, but additionally takes into account the contributions from the lower resonances $\rho, \omega$ and $\phi$. This adequately describes the distribution in the $q^{2}$ bin, around $1 \mathrm{GeV}^{2}$.

With the steadily improving lattice calculations for the various input hadronic quantities and the form factors, theoretical error indicated in Eq. (24) will go down considerably. Experimentally, we expect rapid progress due to the increased statistics at the LHC, but also from Belle II, which will measure the corresponding distributions and branching ratio also in the decays $B^{+} \rightarrow \pi^{+} e^{+} e^{-}$, and $B^{+} \rightarrow \pi^{+} \tau^{+} \tau^{-}$, providing a complementary test of the $e-\mu-\tau$ universality in $b \rightarrow d$ semileptonic transitions.

\section{Leptonic Rare $B$ Decays}

The final topic discussed in this write-up involves purely leptonic decays $B_{s}^{0} \rightarrow \ell^{+} \ell^{-}$ and $B^{0} \rightarrow \ell^{+} \ell^{-}$with $\ell^{+} \ell^{-}=e^{+} e^{-}, \mu^{+} \mu^{-}, \tau^{+} \tau^{-}$. Of these, $\mathcal{B}\left(B_{s}^{0} \rightarrow \mu^{+} \mu^{-}\right)=$ $\left(2.8_{-0.6}^{+0.7}\right) \times 10^{-9}$ is now well measured, and the corresponding CKM-suppressed decay $\mathcal{B}\left(B^{0} \rightarrow \mu^{+} \mu^{-}\right)=\left(3.9_{-1.4}^{+1,6}\right) \times 10^{-10}$ is almost on the verge of becoming a measurement. These numbers are from the combined CMS/LHCb data ${ }^{83}$. From the experimental point of view, their measurement is a real tour de force, considering the tiny branching ratios and the formidable background at the LHC.

In the SM, these decays are dominated by the axial-vector operator $O_{10}=$ $\left(\bar{s}_{\alpha} \gamma^{\mu} P_{L} b_{\alpha}\right)\left(\bar{\ell} \gamma_{\mu} \gamma_{5} \ell\right)$. In principle, the operators $O_{S}=m_{b}\left(\bar{s}_{\alpha} \gamma^{\mu} P_{R} b_{\alpha}\right)(\bar{\ell} \ell)$ and $O_{P}=m_{b}\left(\bar{s}_{\alpha} \gamma^{\mu} P_{R} b_{\alpha}\right)\left(\bar{\ell} \gamma_{5} \ell\right)$ also contribute, but are chirally suppressed in the SM. This need not be the case in BSM scenarios, and hence the great interest in measuring these decays precisely. In the SM, the measurement of $\mathcal{B}\left(B_{s}^{0} \rightarrow \mu^{+} \mu^{-}\right)$and $\mathcal{B}\left(B^{0} \rightarrow \mu^{+} \mu^{-}\right)$provide a measurement of the Wilson coefficient $C_{10}\left(m_{b}\right)$. Their ratio $\mathcal{B}\left(B^{0} \rightarrow \mu^{+} \mu^{-}\right) / \mathcal{B}\left(B_{s}^{0} \rightarrow \mu^{+} \mu^{-}\right)$being proportional to the ratio of the CKM matrix-elements $\left|V_{t d} / V_{t s}\right|^{2}$ is an important constraint on the CKM unitarity triangle. 


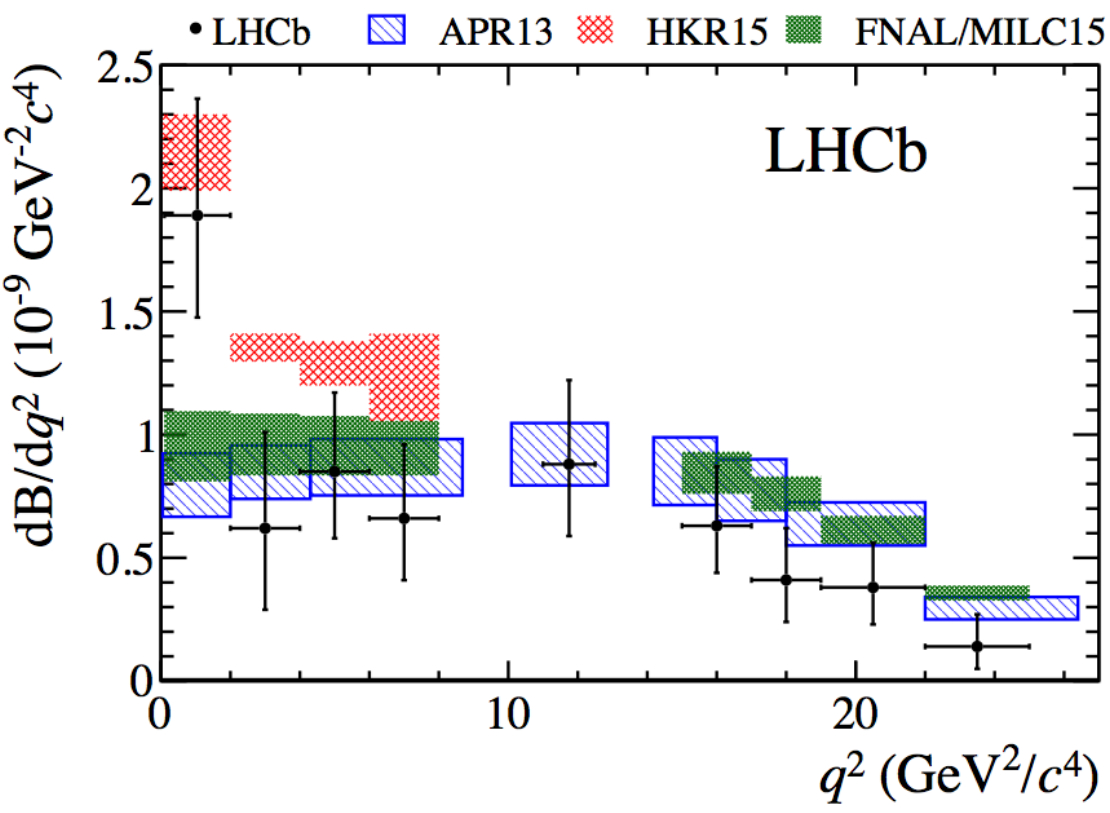

Fig. 11. Comparison of the dimuon invariant mass distribution in $B^{+} \rightarrow \pi^{+} \mu^{+} \mu^{-}$in the SM with the LHCb data $\frac{82}{82}$. Theoretical distributions shown are : APR13 $\sqrt{78}$, HKR15 $\sqrt{81}$, and FNAL/MILC 56 .

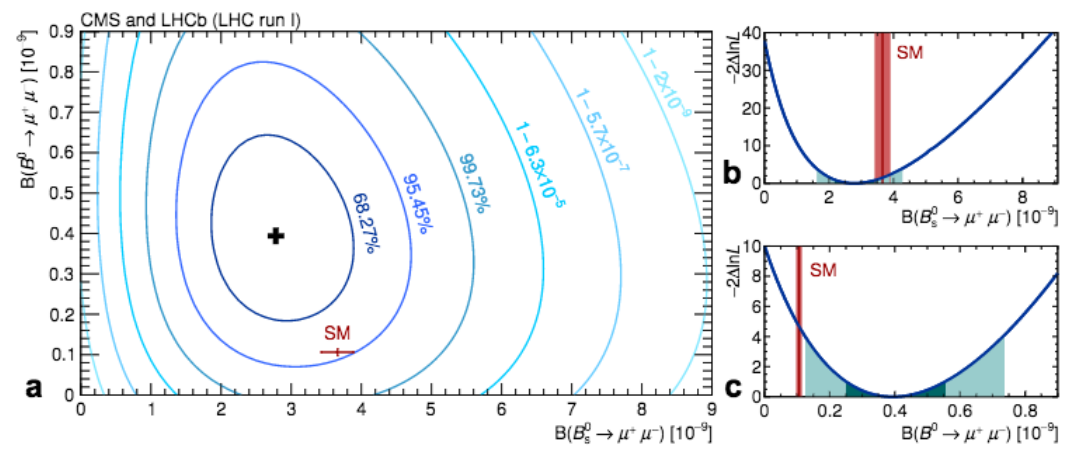

Fig. 12. Likelihood contours in the $\mathcal{B}\left(B^{0} \rightarrow \mu^{+} \mu^{-}\right)$versus $\mathcal{B}\left(B_{s}^{0} \rightarrow \mu^{+} \mu^{-}\right)$plane. The (black) cross in (a) marks the best-fit value and the SM expectation is shown as the (red) marker. Variations of the test statistics $-2 \Delta \ln L$ for $\mathcal{B}\left(B_{s}^{0} \rightarrow \mu^{+} \mu^{-}\right)$(b) and $\mathcal{B}\left(B^{0} \rightarrow \mu^{+} \mu^{-}\right)$(c) are shown. The SM prediction is denoted with the vertical (red) bars. (From the combined CMS-LHCb data 83 !)

The decay rate $\Gamma\left(B_{s}^{0} \rightarrow \mu^{+} \mu^{-}\right)$in the $\mathrm{SM}$ can be written as

$$
\Gamma\left(B_{s}^{0} \rightarrow \mu^{+} \mu^{-}\right)=\frac{G_{F}^{2} M_{W}^{2} m_{B_{s}}^{3} f_{B_{s}}^{2}}{8 \pi^{5}}\left|V_{t b}^{*} V_{t s}\right|^{2} \frac{4 m_{\ell}^{2}}{m_{B_{s}}^{2}} \sqrt{1-\frac{4 m_{\ell}^{2}}{m_{B_{s}}^{2}}}\left|C_{10}\right|^{2}+O\left(\alpha_{\mathrm{em}}\right) .
$$


The coefficient $C_{10}$ has been calculated by taking into account the NNLO QCD corrections and NLO electroweak corrections, but the $O\left(\alpha_{\mathrm{em}}\right)$ contribution indicated above is ignored, as it is small. The SM branching ratio in this accuracy have been obtained in $\frac{84}{86}$, where a careful account of the various input quantities is presented. The importance of including the effects of the width difference $\Delta \Gamma_{s}$ due to the $B_{s}^{0}-\bar{B}_{s}^{0}$ mixings in extracting the branching ratio for $B_{s} \rightarrow \mu^{+} \mu^{-}$has been emphasised in the literature ${ }^{87}$ and is included in the analysis. The timeaveraged branching ratios, which in the SM to a good approximation equals to $\overline{\mathcal{B}}\left(B_{s} \rightarrow \mu^{+} \mu^{-}\right)=\Gamma\left(B_{s} \rightarrow \mu^{+} \mu^{-}\right) / \Gamma_{H}\left(B_{s}\right)$, where $\Gamma_{H}\left(B_{s}\right)$ is the heavier masseigenstate total width, is given below 84

$$
\overline{\mathcal{B}}\left(B_{s} \rightarrow \mu^{+} \mu^{-}\right)=(3.65 \pm 0.23) \times 10^{-9} .
$$

In evaluating this, a value $f_{B_{s}}=227.7(4.5) \mathrm{MeV}$ was used from the earlier FLAG average $^{88}$. In the most recent compilation by the FLAG collaboration ${ }^{9}$, this coupling constant has been updated to $f_{B_{s}}=224(5) \mathrm{MeV}$, which reduces the branching ratio to $\overline{\mathcal{B}}\left(B_{s} \rightarrow \mu^{+} \mu^{-}\right)=(3.55 \pm 0.23) \times 10^{-9}$. This is compatible with the current measurements to about $1 \sigma$, with the uncertainty dominated by the experiment.

The corresponding branching ratio $\overline{\mathcal{B}}\left(B^{0} \rightarrow \mu^{+} \mu^{-}\right)$is evaluated as 84

$$
\overline{\mathcal{B}}\left(B^{0} \rightarrow \mu^{+} \mu^{-}\right)=(1.06 \pm 0.09) \times 10^{-10},
$$

which, likewise, has to be scaled down to $(1.01 \pm 0.09) \times 10^{-10}$, due to the current average $^{9} f_{B}=186(4) \mathrm{MeV}$, compared to $f_{B}=190.5(4.2) \mathrm{MeV}$ used in deriving the result given in Eq. 28). This is about $2 \sigma$ below the current measurement, and the ratio of the two leptonic decays $\overline{\mathcal{B}}\left(B_{s} \rightarrow \mu^{+} \mu^{-}\right) / \overline{\mathcal{B}}\left(B^{0} \rightarrow \mu^{+} \mu^{-}\right)$is off by about $2.3 \sigma$. The likelihood contours in the $\mathcal{B}\left(B^{0} \rightarrow \mu^{+} \mu^{-}\right)$versus $\mathcal{B}\left(B_{s}^{0} \rightarrow \mu^{+} \mu^{-}\right)$plane from the combined CMS/LHCb data are shown in Fig. 12

The anomalies in the decays $B \rightarrow K^{*} \mu^{+} \mu^{-}$, discussed previously, and the deviations in $\mathcal{B}\left(B^{0} \rightarrow \mu^{+} \mu^{-}\right)$and $\mathcal{B}\left(B_{s}^{0} \rightarrow \mu^{+} \mu^{-}\right)$, if consolidated experimentally, would require an extension of the SM. A recent proposal based on the group $S U(3)_{C} \times S U(3)_{L} \times U(1)$ is discussed by Buras, De Fazio and Girrbach $\frac{89}{\text {. Lepton }}$ non-universality, if confirmed, requires a leptoquark-type solution. A viable candidate theory to replace the SM and accounting for all the current anomalies, in my opinion, is not in sight.

\section{Global fits of the Wilson Coefficients $C_{9}$ and $C_{10}$}

As discussed in the foregoing, a number of deviations from the SM-estimates are currently present in the data on semileptonic and leptonic rare $B$-decays. They lie mostly around 2 to $3 \sigma$. A comparison of the $\mathrm{LHCb}$ data on a number of angular observables $F_{\mathrm{L}}, A_{\mathrm{FB}}, S_{3}, \ldots, S_{9}$ in $B^{0} \rightarrow K^{* 0}\left(\rightarrow K^{+} \pi^{-}\right) \mu^{+} \mu^{-}$with the SM-based estimates was shown in Fig, 10 , yielding a value of $\operatorname{Re}\left(C_{9}\right)$ which deviates from the SM by about $3 \sigma$. A number of groups has undertaken similar fits of the data and the outcome depends on a number of assumed correlations. However, it should be 
stressed that there are still non-perturbative contributions present in the current theoretical estimates which are not yet under complete quantitative control. The contributions from the charm quarks in the loops is a case in point. Also, form factor uncertainties are probably larger than assumed in some of these global fits.

As a representative example of the kind of constraints on the Wilson coefficients $C_{9}$ and $C_{10}$ that follow from the data on semileptonic and leptonic decays of the $B$ mesons is shown in Fig. 13 from the Fermilab/MILC collaboration ${ }^{56}$. This shows that the SM point indicated by $(0,0)$ in the $\operatorname{Re}\left(C_{9}^{N P}, \operatorname{Re}\left(C_{10}^{N P}\right)\right.$-plane lies a little beyond $2 \sigma$. In some other fits, the deviations are larger but still far short for a discovery of BSM effects. As a lot of the experimental input in this and similar analysis is due to the $\mathrm{LHCb}$ data, this has to be confirmed by an independent experiment. This, hopefully, will be done by Belle II. We are better advised to wait and see if these deviations become statistically significant enough to warrant new physics. Currently, the situation is tantalising but not conclusive.
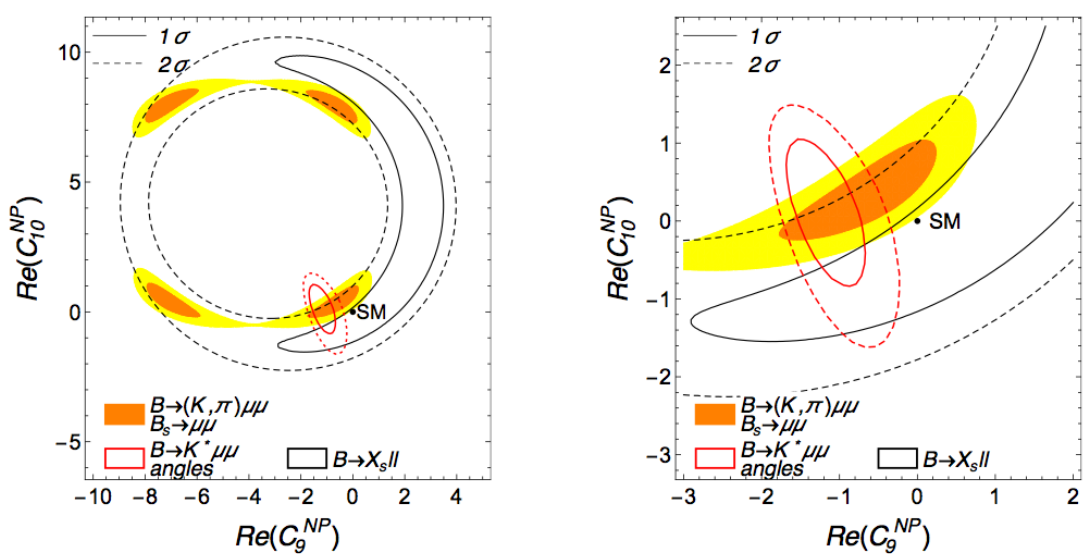

Fig. 13. Present constraints on the Wilson coefficients $\operatorname{Re} C_{10}^{\mathrm{NP}}$ vs. $\operatorname{Re} C_{9}^{\mathrm{NP}}$ from the semileptonic rare $B$-decays and $B_{s} \rightarrow \mu^{+} \mu^{-}$. The SM-point is indicated. (From Fermilab/MILC Lattice Collaboration $[56$.)

\section{Concluding Remarks}

From the measurement by the CLEO collaboration of the rare decay $B \rightarrow X_{s} \gamma$ in 1995 , having a branching ratio of about $3 \times 10^{-4}$, to the rarest of the measured $B$ decays, $B^{0} \rightarrow \mu^{+} \mu^{-}$, with a branching fraction of about $1 \times 10^{-10}$ by the LHCb and CMS collaborations, SM has been tested over six orders of magnitude. This is an impressive feat, made possible by dedicated experimental programmes carried out with diverse beams and detection techniques over a period of more than 20 years. A sustained theoretical effort has accompanied the experiments all along, underscoring both the continued theoretical interest in $b$ physics and an intense exchange between 
the two communities. With the exception of a few anomalies, showing deviations from the SM ranging between 2 to $4 \sigma$ in statistical significance, a vast majority of the measurements is in quantitative agreement with the SM. In particular, all quark flavour transitions are described by the CKM matrix whose elements are now determined. The CP asymmetry measured so far in laboratory experiments is explained by the Kobayashi-Maskawa phase. FCNC processes, of which rare $B$ decays discussed here is a class, are governed by the GIM mechanism, with the particles in the SM (three families of quarks and leptons, electroweak gauge bosons, gluons, and the Higgs) accounting for all the observed phenomena - so far. Whether this astounding consistence will continue will be tested in the coming years, as the LHC experiments analyse more data, enabling vastly improved precision in some of the key measurements discussed here. In a couple of years from now, Belle II will start taking data, providing independent and new measurements. They will be decisive in either deepening our knowledge about the SM, or hopefully in discovering the new frontier of physics.

\section{Acknowledgment}

I thank Harald Fritzsch for inviting me to this very stimulating conference and Prof. K.K. Phua for the warm hospitality in Singapore. I also thank Mikolaj Misiak for reading the manuscript and helpful suggestions.

\section{References}

1. S. L. Glashow, Nucl. Phys. 22, 579 (1961); S. Weinberg, Phys. Rev. Lett. 19, 1264 (1967); A. Salam, Conf. Proc. C 680519, 367 (1968).

2. S. L. Glashow, J. Iliopoulos and L. Maiani, Phys. Rev. D 2, 1285 (1970).

3. N. Cabibbo, Phys. Rev. Lett. 10, 531 (1963). M. Kobayashi and T. Maskawa, Prog. Theor. Phys. 49, 652 (1973).

4. K. A. Olive et al. [Particle Data Group Collaboration], Chin. Phys. C 38, 090001 (2014).

5. A. V. Manohar and M. B. Wise, Camb. Monogr. Part. Phys. Nucl. Phys. Cosmol. 10, $1(2000)$.

6. C. W. Bauer, S. Fleming, D. Pirjol and I. W. Stewart, Phys. Rev. D 63, 114020 (2001) hep-ph/0011336] C. W. Bauer, S. Fleming and M. E. Luke, Phys. Rev. D 63, 014006 (2000) hep-ph/0005275]; C. W. Bauer, D. Pirjol and I. W. Stewart, Phys. Rev. D 65, 054022 (2002) hep-ph/0109045].

7. M. Beneke, A. P. Chapovsky, M. Diehl and T. Feldmann, Nucl. Phys. B 643, 431 (2002) hep-ph/0206152.

8. For an excellent review on SCET and applications, see T. Becher, A. Broggio and A. Ferroglia, Lect. Notes Phys. 896 (2015) arXiv:1410.1892 [hep-ph]].

9. S. Aoki et al., arXiv:1607.00299 [hep-lat].

10. A. Ali, V. M. Braun and H. Simma, Z. Phys. C 63, 437 (1994) hep-ph/9401277.

11. P. Colangelo and A. Khodjamirian, In *Shifman, M. (ed.): At the frontier of particle physics, vol. 3* 1495-1576 hep-ph/0010175].

12. P. Ball and R. Zwicky, Phys. Rev. D 71, 014015 (2005) hep-ph/0406232.

13. A. Bharucha, D. M. Straub and R. Zwicky, arXiv:1503.05534 [hep-ph]. 
14. R. Ammar et al. [CLEO Collaboration], Phys. Rev. Lett. 71, 674 (1993).

15. M. S. Alam et al. [CLEO Collaboration], Phys. Rev. Lett. 74, 2885 (1995).

16. A. Ali and C. Greub, Phys. Lett. B 259, 182 (1991); Z. Phys. C 49, 431 (1991).

17. S. Chen et al. [CLEO Collaboration], Phys. Rev. Lett. 87, 251807 (2001) hepex/0108032

18. P. Koppenburg et al. [Belle Collaboration], Phys. Rev. Lett. 93, 061803 (2004) hepex/0403004.

19. T. Blake, G. Lanfranchi and D. M. Straub, arXiv:1606.00916 [hep-ph].

20. S. Descotes-Genon, L. Hofer, J. Matias and J. Virto, JHEP 1606, 092 (2016) arXiv:1510.04239 [hep-ph]].

21. P. Koppenburg, Z. Dolezal and M. Smizanska, Scholarpedia 11, 32643 (2016) arXiv:1606.00999 [hep-ex]].

22. The physics of the B factories is excellently reviewed in A. J. Bevan et al. [BaBar and Belle Collaborations], Eur. Phys. J. C 74, 3026 (2014) arXiv:1406.6311 [hep-ex]].

23. Y. Amhis et al. [Heavy Flavor Averaging Group (HFAG) Collaboration], arXiv:1412.7515 [hep-ex].

24. M. Misiak et al., Phys. Rev. Lett. 114, no. 22, 221801 (2015) arXiv:1503.01789 [hep$\mathrm{ph}]]$.

25. M. Czakon, P. Fiedler, T. Huber, M. Misiak, T. Schutzmeier and M. Steinhauser, JHEP 1504, 168 (2015) arXiv:1503.01791 [hep-ph]].

26. M. Benzke, S. J. Lee, M. Neubert and G. Paz, JHEP 1008, 099 (2010) arXiv:1003.5012 [hep-ph]]

27. M. Misiak et al., Phys. Rev. Lett. 98, 022002 (2007) hep-ph/0609232.

28. T. Hermann, M. Misiak and M. Steinhauser, JHEP 1211, 036 (2012) arXiv:1208.2788 [hep-ph]]

29. A. Khodjamirian, T. Mannel, A. A. Pivovarov and Y.-M. Wang, JHEP 1009, 089 (2010) arXiv:1006.4945 [hep-ph]].

30. M. Beneke, G. Buchalla, M. Neubert and C. T. Sachrajda, Phys. Rev. Lett. 83 (1999) 1914 hep-ph/9905312.

31. M. Beneke, T. Feldmann and D. Seidel, Nucl. Phys. B 612, 25 (2001) hep$\mathrm{ph} / 0106067$.

32. A. Ali and A. Y. Parkhomenko, Eur. Phys. J. C 23, 89 (2002) hep-ph/0105302.

33. S. W. Bosch and G. Buchalla, Nucl. Phys. B 621, 459 (2002) hep-ph/0106081.

34. M. Beneke, T. Feldmann and D. Seidel, Eur. Phys. J. C 41, 173 (2005) hep$\mathrm{ph} / 0412400$.

35. H. n. Li and H. L. Yu, Phys. Rev. D 53, 2480 (1996) hep-ph/9411308.

36. Y. Y. Keum, H. N. Li and A. I. Sanda, Phys. Rev. D 63, 054008 (2001) hep$\mathrm{ph} / 0004173$

37. Y. Y. Keum, M. Matsumori and A. I. Sanda, Phys. Rev. D 72, 014013 (2005) hep$\mathrm{ph} / 0406055$.

38. C. D. Lu, M. Matsumori, A. I. Sanda and M. Z. Yang, Phys. Rev. D 72, 094005 (2005) Erratum: [Phys. Rev. D 73, 039902 (2006)] hep-ph/0508300].

39. J. g. Chay and C. Kim, Phys. Rev. D 68, 034013 (2003) hep-ph/0305033.

40. T. Becher, R. J. Hill and M. Neubert, Phys. Rev. D 72, 094017 (2005) hep$\mathrm{ph} / 0503263$

41. A. Ali, B. D. Pecjak and C. Greub, Eur. Phys. J. C 55, 577 (2008) arXiv:0709.4422 [hep-ph]].

42. A. Ghinculov, T. Hurth, G. Isidori and Y. P. Yao, Nucl. Phys. B 685, 351 (2004) hep-ph/0312128.

43. H. H. Asatryan, H. M. Asatrian, C. Greub and M. Walker, Phys. Rev. D 65, 074004 
(2002) hep-ph/0109140.

44. A. Ali, E. Lunghi, C. Greub and G. Hiller, Phys. Rev. D 66, 034002 (2002) hepph/0112300.

45. T. Huber, E. Lunghi, M. Misiak and D. Wyler, Nucl. Phys. B 740, 105 (2006) hepph/0512066

46. T. Huber, T. Hurth and E. Lunghi, Nucl. Phys. B 802, 40 (2008) arXiv:0712.3009 [hep-ph]].

47. A. Ali, T. Mannel and T. Morozumi, Phys. Lett. B 273, 505 (1991).

48. F. Kruger and L. M. Sehgal, Phys. Lett. B 380, 199 (1996) hep-ph/9603237.

49. T. Huber, T. Hurth and E. Lunghi, JHEP 1506, 176 (2015) arXiv:1503.04849 [hep$\mathrm{ph}]]$.

50. J. P. Lees et al. [BaBar Collaboration], Phys. Rev. Lett. 112, 211802 (2014) arXiv:1312.5364 [hep-ex]].

51. Y. Sato et al. [Belle Collaboration], Phys. Rev. D 93, no. 3, 032008 (2016) Addendum: [Phys. Rev. D 93, no. 5, 059901 (2016)] arXiv:1402.7134 [hep-ex]].

52. A. Bharucha, T. Feldmann and M. Wick, JHEP 1009, 090 (2010) arXiv:1004.3249 [hep-ph]].

53. E. Dalgic, A. Gray, M. Wingate, C. T. H. Davies, G. P. Lepage and J. Shigemitsu, Phys. Rev. D 73, 074502 (2006) Erratum: [Phys. Rev. D 75, 119906 (2007)] heplat/0601021.

54. C. M. Bouchard, G. P. Lepage, C. J. Monahan, H. Na and J. Shigemitsu, PoS LATTICE 2013, 387 (2014) arXiv:1310.3207 [hep-lat]].

55. C. Bouchard et al. [HPQCD Collaboration], Phys. Rev. D 88, no. 5, 054509 (2013) Erratum: [Phys. Rev. D 88, no. 7, 079901 (2013)] arXiv:1306.2384 [hep-lat]].

56. J. A. Bailey et al. [Fermilab Lattice and MILC Collaborations], Phys. Rev. Lett. 115, no. 15, 152002 (2015) arXiv:1507.01618 [hep-ph]].

57. M. Beneke and T. Feldmann, Nucl. Phys. B 592, 3 (2001) hep-ph/0008255.

58. A. Ali, G. Kramer and G. h. Zhu, Eur. Phys. J. C 47, 625 (2006) hep-ph/0601034.

59. R. Aaij et al. [LHCb Collaboration], Phys. Rev. Lett. 113, 151601 (2014) arXiv:1406.6482 [hep-ex]].

60. C. Bobeth, G. Hiller and G. Piranishvili, JHEP 0712, 040 (2007) arXiv:0709.4174 [hep-ph]].

61. C. W. Chiang, X. G. He and G. Valencia, Phys. Rev. D 93, no. 7, 074003 (2016) arXiv:1601.07328 [hep-ph]].

62. G. Hiller and M. Schmaltz, Phys. Rev. D 90, 054014 (2014) arXiv:1408.1627[hep-ph]].

63. M. Bauer and M. Neubert, Phys. Rev. Lett. 116, no. 14, 141802 (2016) arXiv:1511.01900 [hep-ph]].

64. R. Barbieri, G. Isidori, A. Pattori and F. Senia, Eur. Phys. J. C 76, no. 2, 67 (2016) arXiv:1512.01560 [hep-ph]].

65. J. C. Pati and A. Salam, Phys. Rev. D 8, 1240 (1973).

66. J. C. Pati and A. Salam, Phys. Rev. D 10, 275 (1974) Erratum: [Phys. Rev. D 11, $703(1975)]$.

67. I. Dorsner, S. Fajfer, A. Greljo, J. F. Kamenik and N. Kosnik, arXiv:1603.04993 [hep$\mathrm{ph}$.

68. R. Aaij et al. [LHCb Collaboration], JHEP 1602, 104 (2016) arXiv:1512.04442 [hepex]].

69. R. Aaij et al. [LHCb Collaboration], JHEP 1307, 084 (2013) arXiv:1305.2168 [hepex]].

70. S. Descotes-Genon, T. Hurth, J. Matias and J. Virto, JHEP 1305, 137 (2013) arXiv:1303.5794 [hep-ph]]. 
71. S. Jäger and J. Martin Camalich, JHEP 1305, 043 (2013) arXiv:1212.2263 [hep-ph]].

72. S. Jäger and J. Martin Camalich, Phys. Rev. D 93, no. 1, 014028 (2016) arXiv:1412.3183 [hep-ph]].

73. W. Altmannshofer and D. M. Straub, Eur. Phys. J. C 75, no. 8, 382 (2015) arXiv:1411.3161 [hep-ph]].

74. T. Hurth and F. Mahmoudi, JHEP 1404, 097 (2014) arXiv:1312.5267[hep-ph]].

75. S. Descotes-Genon, L. Hofer, J. Matias and J. Virto, JHEP 1412, 125 (2014) arXiv:1407.8526 [hep-ph]].

76. A. Abdesselam et al. [Belle Collaboration], arXiv:1604.04042 [hep-ex].

77. M. Ciuchini, M. Fedele, E. Franco, S. Mishima, A. Paul, L. Silvestrini and M. Valli, JHEP 1606, 116 (2016) arXiv:1512.07157 [hep-ph]].

78. A. Ali, A. Y. Parkhomenko and A. V. Rusov, Phys. Rev. D 89, no. 9, 094021 (2014) arXiv:1312.2523 [hep-ph]].

79. C. G. Boyd, B. Grinstein and R. F. Lebed, Phys. Rev. Lett. 74, 4603 (1995) hepph/9412324.

80. C. Bourrely, I. Caprini and L. Lellouch, Phys. Rev. D 79, 013008 (2009) Erratum: [Phys. Rev. D 82, 099902 (2010)] arXiv:0807.2722 [hep-ph]].

81. C. Hambrock, A. Khodjamirian and A. Rusov, Phys. Rev. D 92, no. 7, 074020 (2015) arXiv:1506.07760 [hep-ph]].

82. R. Aaij et al. [LHCb Collaboration], JHEP 1510 (2015) 034 arXiv:1509.00414 [hepex]].

83. V. Khachatryan et al. [CMS and LHCb Collaborations], Nature 522, 68 (2015) arXiv:1411.4413 [hep-ex]].

84. C. Bobeth, M. Gorbahn, T. Hermann, M. Misiak, E. Stamou and M. Steinhauser, Phys. Rev. Lett. 112, 101801 (2014) arXiv:1311.0903 [hep-ph]].

85. T. Hermann, M. Misiak and M. Steinhauser, JHEP 1312, 097 (2013) arXiv:1311.1347 [hep-ph]].

86. C. Bobeth, M. Gorbahn and E. Stamou, Phys. Rev. D 89, no. 3, 034023 (2014) arXiv:1311.1348 [hep-ph]].

87. K. De Bruyn, R. Fleischer, R. Knegjens, P. Koppenburg, M. Merk, A. Pellegrino and N. Tuning, Phys. Rev. Lett. 109, 041801 (2012) arXiv:1204.1737[hep-ph]].

88. S. Aoki et al., Eur. Phys. J. C 74, 2890 (2014) arXiv:1310.8555 [hep-lat]].

89. A. J. Buras, F. De Fazio and J. Girrbach, JHEP 1402, 112 (2014) arXiv:1311.6729 [hep-ph]]. 